\title{
NCAM-Induced Neurite Outgrowth Depends on Binding of Calmodulin to NCAM and on Nuclear Import of NCAM and fak Fragments
}

\author{
Ralf Kleene, ${ }^{1 \star}$ Mounir Mzoughi, ${ }^{1 \star}$ Gunjan Joshi, ${ }^{1}$ Ina Kalus, ${ }^{1}$ Ulrich Bormann, ${ }^{1}$ Christian Schulze, ${ }^{1}$ Mei-Fang Xiao, ${ }^{1}$ \\ Alexander Dityatev, ${ }^{1}$ and Melitta Schachner ${ }^{1,2,3}$ \\ ${ }^{1}$ Zentrum für Molekulare Neurobiologie, Universitätsklinikum Hamburg-Eppendorf, 20246 Hamburg, Germany, and ${ }^{2}$ Keck Center for Collaborative \\ Neuroscience and ${ }^{3}$ Department of Cell Biology and Neuroscience, Rutgers University, Piscataway, New Jersey 08854
}

\begin{abstract}
The neural cell adhesion molecule NCAM plays important functional roles not only during nervous system development, but also in the adult after injury and in synaptic plasticity. Homophilic binding of NCAM triggers intracellular signaling events resulting in cellular responses such as neurite outgrowth that require NCAM palmitoylation-dependent raft localization and activation of the nonreceptor tyrosine kinases fyn and fak. In this study, we show that stimulation of NCAM by a function-triggering NCAM antibody results in proteolytic processing of NCAM and fak. The C-terminal fragment of NCAM, consisting of the intracellular domain, the transmembrane domain, and a stub of the extracellular domain, and the N-terminal fragment of fak are imported into the nucleus. NCAM-stimulated fak activation, generation, and nuclear import of NCAM and fak fragments as well as neurite outgrowth are abolished by mutation of the calmodulin binding motif in the intracellular domain of NCAM that is responsible for the calcium-dependent binding of calmodulin to NCAM. This mutation interferes neither with NCAM cell surface expression, palmitoylation, and raft localization nor with fyn activation. The way by which the transmembrane NCAM fragment reaches the nucleus in a calmodulin- and calcium-dependent manner is by endocytotic transport via the endoplasmic reticulum and the cytoplasm. The generation and nuclear import of NCAM and phosphorylated fak fragments resulting from NCAM stimulation may represent a signal pathway activating cellular responses in parallel or in association with classical kinase- and phosphorylation-dependent signaling cascades.
\end{abstract}

\section{Introduction}

Transmembrane adhesion molecules not only mediate recognition between cells, but also transduce signals to the cell interior to elicit cellular responses (Maness and Schachner, 2007; Ditlevsen et al., 2008) regulating ontogenetic development, synaptic plasticity, and regeneration in the adult. The neural cell adhesion molecule (NCAM) is an evolutionarily conserved Ig superfamily adhesion molecule first discovered in the nervous system (Cunningham et al., 1983). The three major isoforms of NCAM, called NCAM180, NCAM140, and NCAM120, are generated from a single gene by alternative splicing of a primary transcript. NCAM180 and NCAM140 are transmembrane isoforms, while NCAM120 is attached to the membrane via a glycophosphatidylinositol anchor. Functional triggering of NCAM

\footnotetext{
Received Jan. 19, 2010; revised May 26, 2010; accepted June 27, 2010.

M.S. is supported by the commission for Spinal Cord Research and is consultant at the Center for Neuroscience at Shantou University Medical College, China. We are grateful to Galina Dityateva for help with transfection of hippocampal neurons, Ute Bork for excellent technical assistance, Eva Kronberg for excellent animal care, Christo Goridis (Département de Biologie, Ecole Normale Supérieure, Paris, France) for the P61 antibody, and Patricia Maness (University of North Carolina, Chapel Hill, NC) for the expression vectors pCDNA3-NCAM140 and pCDNA3-NCAM180. We are grateful to Claus Schafer-Nielsen (Schafer-N, Copenhagen, Denmark) and Jacob Zij)stra (Novartis Consumer Health, Nyon, Switzerland) for synthetic peptides and the calmodulin inhibitor CGS9343B, respectively.

${ }^{*}$ R.K. and M.M. contributed equally to this work.

Correspondence should be addressed to either Melitta Schachner or Ralf Kleene, Zentrum für Molekulare Neurobiologie, Universitätsklinikum Hamburg-Eppendorf, Martinistrasse 52, 20246 Hamburg, Germany, E-mail: melitta. schachner@zmnh.uni-hamburg.de or ralf.kleene@zmnh.uni-hamburg.de.

D01:10.1523/JNEUROSCI.0297-10.2010

Copyright $\odot 2010$ the authors $\quad 0270-6474 / 10 / 3010784-15 \$ 15.00 / 0$
}

leads to neural cell migration, neurite outgrowth and fasciculation, synaptogenesis, synaptic plasticity, and certain forms of emotional behavior, thus most likely implicating different molecular mechanisms in signaling (for reviews, see Brennaman and Maness, 2010; Conboy et al., 2010; Ditlevsen and Kolkova, 2010; Muller et al., 2010). Homophilic or heterophilic interactions between NCAM and its extracellular binding partners, in particular with fibroblast growth factor (FGF) receptors (for review, see Kiselyov, 2010) and glial cell line-derived neurotrophic factor (GDNF) receptor (Paratcha et al., 2003), trigger NCAMdependent intracellular signaling events resulting in these distinct cellular responses. NCAM-dependent neurite outgrowth requires the activation of the nonreceptor tyrosine kinase fyn (Beggs et al., 1994; Kolkova et al., 2000), which interacts with NCAM140 and is activated by NCAM stimulation (Beggs et al., 1997; Bodrikov et al., 2005). Fyn activation triggers the focal adhesion kinase fak, which is another nonreceptor tyrosine kinase that associates with NCAM and is also activated upon NCAM stimulation (Beggs et al., 1997; Niethammer et al., 2002). Moreover, NCAM-mediated neurite outgrowth is abolished when cells are transfected with a dominant-negative construct of fak (Kolkova et al., 2000).

Activation of intracellular signaling triggered by NCAM140 depends on NCAM's localization in lipid rafts, which represent specialized microdomains of plasma membranes serving as signaling platforms (for review, see Füllekrug and Simons, 2004), for instance, to trigger neurite outgrowth. Palmitoylation of three 
cysteines in the intracellular domains of the transmembrane NCAM isoforms is required for the recruitment of NCAM to lipid rafts (Little et al., 1998; Niethammer et al., 2002). NCAM's redistribution to lipid rafts is required for activation of fyn and fak (Bodrikov et al., 2005; Ponimaskin et al., 2008).

Here, we show that fak activation depends on the calciumbinding molecule calmodulin for interaction with the intracellular domain of NCAM and that mutation of the calmodulin binding site within NCAM abolishes activation of fak, NCAMstimulated neurite outgrowth, and proteolytic processing of NCAM and activated fak. An unexpected finding indicates that upon NCAM stimulation, a N-terminal fragment of fak and a C-terminal fragment of NCAM containing the transmembrane domain and part of the extracellular domain as well as calmodulin are translocated to the nucleus. Upon NCAM stimulation, the transmembrane NCAM fragment is translocated to the ER and from the ER membrane to the cytoplasm and imported from the cytoplasm to the nucleus in a calmodulin- and calcium-dependent process.

\section{Materials and Methods}

Experimental animals. C57BL/6J mice bred and maintained at the Universitätsklinikum Hamburg-Eppendorf were used for all experiments. NCAM-deficient (NCAM-/-) mice provided by H. Cremer (Developmental Biology Institute of Marseille, Marseille, France) (Cremer et al., 1994) had been backcrossed onto the C57BL/6J background for more than eight generations, and their wild-type $(\mathrm{NCAM}+/+)$ littermates were used as controls. Animals were housed at $25^{\circ} \mathrm{C}$ on a $12 \mathrm{~h}$ light $/ 12 \mathrm{~h}$ dark cycle with ad libitum access to food and water.

Reagents and antibodies. Polyclonal NCAM antibody $1 \beta 2$ or monoclonal NCAM antibody H28, both against the extracellular domain of NCAM, have been described previously (Niethammer et al., 2002). Monoclonal NCAM antibodies P61 or 5B8 against the intracellular domain of NCAM (Gennarini et al., 1984) were obtained from Christo Goridis (Département de Biologie, Ecole Normale Supérieure, Paris, France) or from the Developmental Studies Hybridoma Bank, University of Iowa. Polyclonal L1 and CHL1 antibodies have been described (Rolf et al., 2003). All secondary antibodies were obtained from Dianova. Antibodies directed against calmodulin, fyn, and fak were purchased from Santa Cruz Biotechnology, and the antibody against Tyr-527-dephosphorylated src kinase that cross-reacts with Tyr-531-dephosphorylated fyn was from Cell Signaling Technology. Anti-penta-His antibody was from Qiagen and the sec61 $\beta$ antibody was from Millipore. Antibodies against calreticulin, actin, and heterochromatin-associated protein 1 (HP1) were obtained from Santa Cruz Biotechnology, Sigma-Aldrich, and Cell Signaling Technology, respectively. Primers were from Metabion and synthetic NCAM peptides were from Schafer-N. Protease inhibitors, exotoxin A, trifluoperazine dihydrochloride, and W-7 hydrochloride were from Sigma-Aldrich. The calmodulin inhibitor CGS9343B was a kind gift from Jacob Zijlstra (Novartis Consumer Health, Nyon, Switzerland). NCAM-Fc and immunopurified L1 or NCAM were prepared as described previously (Kalus et al., 2003; Bodrikov et al., 2005; Kalus et al., 2006).

Microexplant cultures, $\mathrm{CHO}$ cell culture, transfection, and pharmacological treatment. Preparation of cerebellar microexplant cultures and quantification of total length of the 10 longest neurites from 10 explants was described previously (Kalus et al., 2003, 2006). CHO cells $\left(10^{5}\right.$ to $10^{6}$ cells/well) were grown on 6-well-plates (Nunc) for $24 \mathrm{~h}$ in DMEM medium containing $10 \%$ fetal calf serum (FCS), 1 mm sodium pyruvate, and antibiotics ( $100 \mathrm{U} / \mathrm{ml}$ penicillin and $100 \mu \mathrm{g} / \mathrm{ml}$ streptomycin). Cells were transfected using Lipofectamine Plus (Invitrogen) according to the manufacturer's instructions. After $24 \mathrm{~h}$, the transfection medium was replaced with serum-free medium and cells were maintained for further $24 \mathrm{~h}$ in the absence or presence of $10 \mu \mathrm{M}$ CGS9343B. Cells and cell culture supernatants were collected separately. Cell culture supernatants were cleared by centrifugation for $1 \mathrm{~h}$ at $100,000 \times g$ at $4^{\circ} \mathrm{C}$. After centrifuga- tion, proteins from the supernatants were concentrated by precipitation (Kalus et al., 2003).

Production and purification of recombinant intracellular domains. Sequences coding for the intracellular domains of NCAM140 (bp 21352550), NCAM180 (bp 2135-2850), CHL1 (bp 3315-3627), and L1 (bp $3438-3780)$ were amplified and restriction sites were introduced by PCR. After restriction digestion the PCR products were cloned into the His-tag-containing $\mathrm{pQE} 30$ plasmid. Protein expression and purification using Ni-NTA beads (Qiagen) were according to the manufacturer's instructions. Bacteria were lysed using a French Press (Spectronic Instruments/SLM Aminco).

Calmodulin binding assays. Agarose beads conjugated with calmodulin from bovine brain $(1 \mu \mathrm{g} / \mu \mathrm{l}$; Calbiochem) were washed twice with RIPA buffer A (50 mм Tris-HCl, pH 8.0, 150 mm NaCl, 1\% Triton X-100, 0.1\% NP-40) and incubated with $10 \mu \mathrm{g}$ of intracellular domains of NCAM140, NCAM180, L1, or CHL1 in $250 \mu \mathrm{l}$ of RIPA buffer supplemented either with $1 \mathrm{~mm} \mathrm{MgCl}_{2}$ and $2 \mathrm{mM} \mathrm{CaCl}_{2}$ or $5 \mathrm{~mm}$ EDTA for $2 \mathrm{~h}$ at $4^{\circ} \mathrm{C}$. Beads were washed 5 times for $5 \mathrm{~min}$ at $4^{\circ} \mathrm{C}$ with RIPA buffer $\mathrm{A}$, boiled in SDS-PAGE sample buffer for $15 \mathrm{~min}$, and centrifuged at $13,000 \times g$ for 5 min at room temperature.

BIACORE surface plasmon resonance analysis was performed as described previously (Makhina et al., 2009). Fifty micrograms per milliliter calmodulin from bovine brain (Sigma) was immobilized on a CM5 sensor chip and recombinant intracellular domains of NCAM, L1, or CHL1 in HEPES buffer (10 mM HEPES, pH 7.4, $150 \mathrm{~mm} \mathrm{NaCl}$ ) supplemented with $1 \mathrm{mM} \mathrm{CaCl}_{2}$ or $5 \mathrm{~mm}$ EDTA were injected.

ELISA, Western blot analysis, immunoprecipitation, and cell surface biotinylation. ELISA experiments, Western blot analysis, and cell surface biotinylation have been described in detail (Makhina et al., 2009). For ELISA, $10-20 \mu \mathrm{g} / \mathrm{ml}$ calmodulin was used for substrate coating. For competition ELISA, a constant amount $(50 \mu \mathrm{g} / \mathrm{ml})$ of intracellular domains of NCAM was preincubated for 10 min with NCAM peptides and then applied to $10-20 \mu \mathrm{g} / \mathrm{ml}$ substrate-coated calmodulin. For immunoprecipitation, cells were washed with PBS, pH 7.3, and lysed in RIPA buffer B (25 mu Tris- $\mathrm{HCl}, 150 \mathrm{~mm} \mathrm{NaCl}, 1 \% \mathrm{NP}-40,1 \%$ sodium deoxycholate, $0.1 \%$ SDS, pH 7.6) and subjected to preclearing and immunoprecipitation using Protein A/G agarose Plus (Santa Cruz Biotechnology). For immunoprecipitation and detection of activated fyn or fak, src antibody that cross-reacts with fyn dephosphorylated at Tyr-531 or anti-phosphotyrosine antibodies were used for immunoprecipitation and fyn- or fak-specific antibodies were used for Western blot analysis, respectively. Beads were then washed with PBS and boiled in SDS-PAGE sample buffer for $5 \mathrm{~min}$ at $95^{\circ} \mathrm{C}$.

Stable transfection of $\mathrm{CHO}$ cells. $\mathrm{CHO}$ cells grown in Glasgow modified Eagle's medium (GMEM) containing 10\% FCS and transfected using Lipofectamine Plus reagent (Invitrogen) according to the manufacturer's instructions. Cells were treated with $0.5 \mathrm{mg} / \mathrm{ml} \mathrm{G} 418$ (Invitrogen) every $72 \mathrm{~h}$ and after 3 weeks, single clones were isolated and grown in medium supplemented with G418.

Palmitoylation assay and isolation of lipid rafts. Stably transfected $\mathrm{CHO}$ cells were maintained in serum-free medium for $16 \mathrm{~h}$ at $37^{\circ} \mathrm{C}$, labeled with $\left[{ }^{3} \mathrm{H}\right.$ ]palmitate $(300 \mu \mathrm{Ci} / \mathrm{ml}, 30-60 \mathrm{Ci} / \mathrm{mmol})$ for $4 \mathrm{~h}$, washed with PBS, and lysed with RIPA buffer C (20 mm Tris-HCl, pH 7.4, $150 \mathrm{~mm}$ $\mathrm{NaCl}, 10$ mм EDTA, 1\% NP-40). After immunoprecipitation of NCAM using polyclonal NCAM antibody and Protein A-Sepharose CL-4B (Sigma), samples were analyzed by SDS-PAGE on $10 \%$ gels under nonreducing conditions. Gels were treated with Amplify solution (GE Healthcare) and subjected to fluorography. Lipid rafts were isolated from stably transfected $\mathrm{CHO}$ cells. Confluent cells from eight $15 \mathrm{~cm}$ dishes were collected using a rubber policeman and pelleted at $700 \times g$ for 10 min at room temperature. Cell pellets were resuspended in four volumes ice-cold 1\% Triton X-100 in Tris-buffered saline, pH 7.3 (TBS), passed through a 27 gauge needle, and incubated on ice for $20 \mathrm{~min}$. The cell lysate was mixed with an equal volume of $80 \%$ sucrose in $0.2 \mathrm{M} \mathrm{Na}_{2} \mathrm{CO}_{3}$ and overlaid with $30 \%$ and $10 \%$ sucrose in TBS. The gradient was centrifuged for $18 \mathrm{~h}$ at $230,000 \times \mathrm{g}$ at $4^{\circ} \mathrm{C}$, and the material from the $10 \% /$ $30 \%$ interphase representing the raft fraction was collected, diluted with TBS, and pelleted at $100,000 \times g$ for $1 \mathrm{~h}$ at $4^{\circ} \mathrm{C}$.

NCAM stimulation and nuclear protein fractionation. For stimulation experiments, $\mathrm{CHO}$ cells or freshly dissociated cerebellar neurons were 
seeded onto 6-well-plates (Nunc) at a density of 190,000 cells/well and serum deprived at a confluence of $80 \%$ for $5 \mathrm{~h}$. CHO cells were stimulated with polyclonal NCAM antibody $1 \beta 2$ (corresponding to $5 \mu \mathrm{g}$ of $\mathrm{IgG} / \mathrm{ml}$ ) for $20 \mathrm{~min}$ at $37^{\circ} \mathrm{C}$. Cerebellar neurons were stimulated with polyclonal NCAM antibody, NCAM-Fc $(10 \mu \mathrm{g} / \mathrm{ml})$, or human Fc $(5 \mu \mathrm{g} / \mathrm{ml})$ in the absence or presence of $10 \mu \mathrm{M}$ DAPT or aprotinin for $1 \mathrm{~h}$ at $37^{\circ} \mathrm{C}$. Qproteome Nuclear Protein Kit (Qiagen) was used for subcellular fractionation of nuclear and non-nuclear compartments according to the manufacturer's instructions.

Cell surface biotinylation and subcellular fractionation of Neuro2a cells and nuclear import assay. Neuro2a cells were grown in Opti-MEM media (Invitrogen) with 10\% FCS in $1915 \mathrm{~cm}$ dishes and serum deprived at a confluence of $80 \%$ for $8-10$ h. For cell surface biotinylation, cells were washed thrice with PBS-2+ (PBS, $0.5 \mathrm{~mm} \mathrm{CaCl}_{2}, 2 \mathrm{mM} \mathrm{MgCl}_{2}$ ) and incubated for $30 \mathrm{~min}$ at room temperature with $0.5 \mathrm{mg} / \mathrm{ml}$ sulfo-NHSLS-biotin (Pierce) in PBS-2+, followed by washing the cells twice with $100 \mathrm{~mm}$ glycine at room temperature. Cells were washed with PBS-2+ and stimulated with either NCAM, L1, or nonimmune control antibodies for $1 \mathrm{~h}$ at $37^{\circ} \mathrm{C}$. The cells were collected using a rubber policeman, centrifuged at $1000 \times g$ and subjected to subcellular fractionation using an ER isolation kit (Sigma) according to the manufacturer's instructions. Briefly, cells were resuspended in hypotonic buffer [100 mM HEPES, pH 7.8, $20 \mathrm{~mm} \mathrm{KCl}$, supplemented without or with $2 \mathrm{~mm}$ EGTA, and 1 protease inhibitor cocktail tablet (Roche Diagnostics) per $50 \mathrm{ml}$ added just before the experiment], incubated for $20 \mathrm{~min}$ on ice, and centrifuged at $600 \times g$ for $5 \mathrm{~min}$. Packed cell volume (PCV) was measured and the pellet was suspended in 2 volumes of isotonic buffer $(50 \mathrm{~mm} \mathrm{HEPES,} \mathrm{pH}$ 7.8, $0.25 \mathrm{~m}$ sucrose, $20 \mathrm{~mm} \mathrm{KCl}$, without or with $2 \mathrm{~mm}$ EGTA, and 1 protease inhibitor cocktail tablet per $50 \mathrm{ml}$ ). The pellet was homogenized using a Dounce homogenizer, passed through a 27 gauge needle several times, and centrifuged at $1000 \times \mathrm{g}$ for $10 \mathrm{~min}$ at $4^{\circ} \mathrm{C}$. The $1000 \times \mathrm{g}$ pellet (nuclear pellet) was saved, the supernatant was centrifuged at 12,000 $\times g$ for $15 \mathrm{~min}$ at $4^{\circ} \mathrm{C}$, and the resulting supernatant was centrifuged at $100,000 \times g$ for $1 \mathrm{~h}$. The supernatant representing the cytoplasmic fraction was saved and the pellet was homogenized in HOMO buffer $(0.32 \mathrm{M}$ sucrose, $10 \mathrm{~mm}$ Tris/HCl, pH 7.4), adjusted to $20 \%$ Optiprep, layered between $30 \%$ and $15 \%$ Optiprep and centrifuged at $150,000 \times g$ for $3 \mathrm{~h}$. The material from the $15 \% / 20 \%$ interphase containing smooth ER (SER) and the material from the $20 \% / 30 \%$ interphase containing rough ER (RER) were collected. For the isolation of nuclei, the $1000 \times g$ nuclear pellet was homogenized in HOMO buffer, applied to a gradient of $35 \%$, $30 \%$, and $25 \%$ Optiprep, and centrifuged at $10,000 \times g$ for $20 \mathrm{~min}$ at $4{ }^{\circ} \mathrm{C}$. Nuclei were collected from the $30 \% / 35 \%$ interphase, diluted two times with $\mathrm{HOMO}$ buffer, and centrifuged at $1000 \times g$ for $20 \mathrm{~min}$ at $4^{\circ} \mathrm{C}$. The pellet was again resuspended in HOMO buffer and centrifuged at $1000 \times$ $g$ for $10 \mathrm{~min}$ at $4^{\circ} \mathrm{C}$. The nuclear pellet was then resuspended in $200 \mu \mathrm{l}$ of extraction buffer (10 mM HEPES, $10 \mathrm{~mm} \mathrm{KCl,} 2 \mathrm{~mm} \mathrm{MgCl}_{2}, 500 \mathrm{~mm}$ $\mathrm{NaCl}, 25 \%$ glycerol, $\mathrm{pH} 7.5,1$ protease inhibitor cocktail tablet per $50 \mathrm{ml}$ ) and incubated on ice for $30 \mathrm{~min}$. After centrifugation at $10,000 \times g$ for 5 $\min$ at $4^{\circ} \mathrm{C}$, the supernatant was collected and the pellet was resuspended in SDS sample buffer and passed through a 27 gauge needle.

For the analysis of translocation of NCAM from the ER membrane to the cytoplasm, the ER fractions were resuspended either in HOMO buffer with or without $100 \mu \mathrm{g} / 200 \mu \mathrm{l}$ calmodulin and incubated with untreated, nonbiotinylated, and nonstimulated cytoplasmic fraction in translocation buffer (10 mM HEPES, 40 mM magnesium acetate, $\mathrm{pH} 7.2$, $1 \mathrm{~mm}$ DTT, $0.1 \mathrm{~mm}$ PMSF) in the absence or presence of $2 \mathrm{~mm} \mathrm{CaCl}_{2}, 2$ mм EGTA, or $2 \mathrm{~mm} \mathrm{CaCl}_{2}$ plus $1 \mathrm{~mm} \mathrm{CGS9343B,} 1 \mu \mathrm{g} / 200 \mu$ l exotoxin A, or $1 \mu \mathrm{l} / 200 \mu \mathrm{l} \mathrm{sec} 61 \beta$ antibody for $60 \mathrm{~min}$ at $37^{\circ} \mathrm{C}$. The samples were then centrifuged at $100,000 \times g$ for $20 \mathrm{~min}$ at $4^{\circ} \mathrm{C}$. The pellets and supernatants were collected and streptavidin-coupled magnetic beads (Invitrogen) were used to isolate biotinylated proteins.

For the analysis of nuclear import, untreated, nonbiotinylated nuclei from cells not stimulated for NCAM were incubated with biotinylated cytoplasmic fraction from NCAM stimulated cells in nuclear translocation buffer (25 mM HEPES, pH 7.4, $12.5 \mathrm{~mm} \mathrm{KCl,} 2.5 \mathrm{mM} \mathrm{MgCl}_{2}, 1.25 \mathrm{~mm}$ $\mathrm{CaCl}_{2}, 0.1 \mathrm{~mm}$ ATP) in the presence of either $2 \mathrm{~mm} \mathrm{CaCl}_{2}$ or $2 \mathrm{~mm}$ EGTA or $2 \mathrm{mM} \mathrm{CaCl}_{2}$ plus $1 \mathrm{~mm}$ CGS9343B. Streptavidin-coupled magnetic beads were used to isolate biotinylated proteins.
For coimmunoprecipitation of calmodulin and biotinylated NCAM fragment, the cytoplasmic fraction was incubated with Protein A/G beads (Santa Cruz Biotechnology) and the calmodulin antibody overnight at $4^{\circ} \mathrm{C}$ after preclearing. The beads were centrifuged at $2000 \times g$ for $5 \mathrm{~min}$, incubated in $1 \%$ SDS for $60 \mathrm{~min}$ at $37^{\circ} \mathrm{C}$. The samples were incubated with streptavidin-coupled magnetic beads overnight at $4^{\circ} \mathrm{C}$ on a headover-head shaker. Beads were then washed with PBS and boiled with SDS sample buffer at $95^{\circ} \mathrm{C}$ for $5 \mathrm{~min}$.

Site-directed mutagenesis. For mutation the QuikChange Site-Directed Mutagenesis Kit (Stratagene) and the primers NCAMcalmut-up (GAC ATC ACC TGC TAC TAC ATG AAC AAG TGT GGC ATG CTC ATG TGC ATC GCT GTT AAC ATG TGT GGC AAA GCT GG) and NCAMcalmut-down (CCA GCT TTG CCA CAC ATG TTA ACA GCG ATG CAC ATG AGC ATG CCA CAC TTG TTC ATG TAG TAG CAG GTG ATG TC) were used according to the manufacturer's instructions.

Primary cultures of dissociated hippocampal and cerebellar neurons and transfection. Primary cultures of cerebellar granule cells were prepared from 6- to 7-d-old C57BL/6J mice as described previously (Loers et al., 2005). Hippocampal neurons were prepared from 1- to 2-d-old C57BL/6J mice and transfected as described previously (Dityateva et al., 2003) using the Nucleofector I kit and program O3 for electroporation (Amaxa). In neurite outgrowth experiments, $10 \mu \mathrm{M}$ CGS9343B, trifluoperazine, and $\mathrm{W}-7$ were used.

Immunocytochemistry of cerebellar neurons and image acquisition. Cultured neurons were washed with ice-cold PBS and fixed in $4 \%$ formaldehyde in PBS for 15 min at room temperature, washed, permeabilized with $1 \%$ Triton X-100 in PBS for 5 min, and blocked in 1\% BSA in PBS for 30 min at $4^{\circ} \mathrm{C}$. Primary antibodies in blocking solution were incubated overnight at $4^{\circ} \mathrm{C}$ and either $\mathrm{Cy} 2-$, Cy3-, or Cy5-conjugated secondary antibodies were incubated for $1 \mathrm{~h}$ in $1 \%$ BSA in PBS at room temperature under exclusion of light. For live-cell staining, the primary NCAM, L1, or CHL1 antibodies were incubated with live cells for $15 \mathrm{~min}$ on ice before fixation. After fixation and washing three times with ice-cold PBS, DRAQ5 (Biostatus Limited) diluted 1:1000 in PBS or the calmodulin antibody was applied for $15 \mathrm{~min}$ at $4^{\circ} \mathrm{C}$. Coverslips were embedded in Fluoromount $\mathrm{G}$ (Southern Biotech). Images of neurons were acquired using an Axiophot 2 microscope (Carl Zeiss) equipped with a digital camera (AxioCam HRc), AxioVision software (version 3.1), and a PlanNeofluar $40 \times$ objective (numerical aperture 0.75 ) or using a confocal laser scanning microscope (LSM510, Carl Zeiss), LSM510 software (version 3 ), and an oil Plan-Neofluar $40 \times$ objective (numerical aperture 1.3) at $3 \times$ digital zoom. Contrast and brightness of the images were further adjusted in Adobe Photoshop CS2.

Statistical analysis. All numerical data are presented as group mean values with SD. Parametric tests ( $t$ test or ANOVA with subsequent Tukey's post hoc test) were used to compare group mean values as appropriate. Data conformed to the requirement for normal distribution ("normality" test, SigmaStat 2.0, SPSS). The threshold value for acceptance of differences between group mean values was $5 \%$.

\section{Results}

\section{NCAM binds to calmodulin in a calcium-dependent manner}

Since proteolytic cleavage of NCAM (Kalus et al., 2006) and L1 (Kalus et al., 2003) is regulated by calmodulin, we investigated whether NCAM or L1 binds directly to calmodulin. The recombinant intracellular domains of NCAM180, NCAM140, L1, and the close homolog of L1 (CHL1) were incubated in the absence or presence of $1 \mathrm{mM}$ calcium with calmodulin immobilized on beads. This high and unphysiological concentration of calcium was used in the binding assays as a first step for verification of the calcium dependence. The intracellular domains of NCAM were pulled down by calmodulin-conjugated beads in the presence, but not in the absence of calcium (Fig. 1A). Calmodulin-conjugated beads did not pull down the intracellular domains of L1 and CHL1, even in the presence of $1 \mathrm{~mm}$ calcium (Fig. $1 \mathrm{~A}$ ).

Binding of the intracellular domains of NCAM180 and NCAM140 to calmodulin was further tested by surface plasmon 


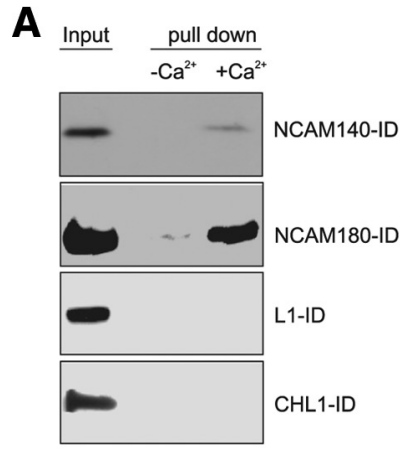

B

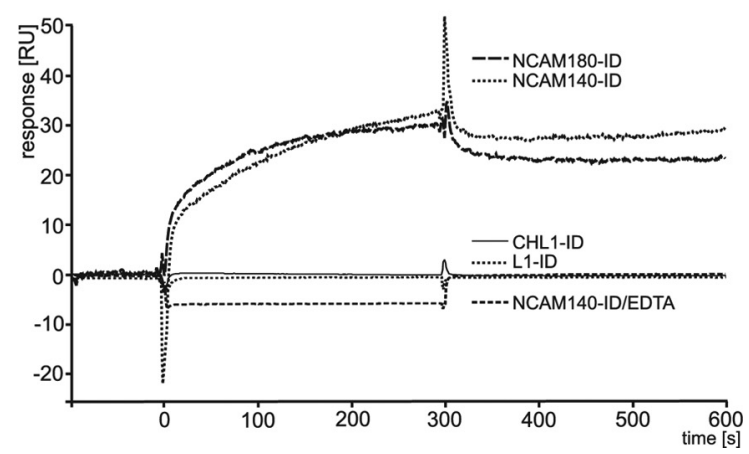

Figure 1. Calmodulin binds to the intracellular domain of NCAM140 and NCAM180 in a calcium-dependent manner. A, Intracellular domains of NCAM140 (NCAM140-ID), NCAM180 (NCAM180-ID), L1 (L1-ID), or CHL1 (CHL1-ID) were incubated with calmodulin immobilized to agarose beads in the absence or presence or $1 \mathrm{mM} \mathrm{CaCl}_{2}$. The beads were pelleted and bound proteins were eluted. The intracellular domains (input) and the eluates (pull down) were subjected to Western blot analysis using an anti-penta His antibody. B, NCAM140-ID, NCAM180-ID, L1-ID, or CHL1-ID were injected over a surface of immobilized calmodulin. Sensorgrams of NCAM140-ID, NCAM180-ID, L1-ID, or CHL1-ID in the presence of $1 \mathrm{~mm} \mathrm{CaCl} 2$ and of NCAM140-ID in the presence of $5 \mathrm{~mm}$ EDTA are shown.

resonance analysis and ELISA. For surface plasmon resonance analysis, calmodulin was immobilized to the sensor surface and the intracellular domains of NCAM180, NCAM140, L1, and CHL1 were injected. The sensorgrams showed binding of the intracellular domains of NCAM140 and NCAM180 to immobilized calmodulin in the presence of $1 \mathrm{mM}$ calcium, but not in the presence of the $5 \mathrm{~mm}$ calcium-chelating agent EDTA (Fig. $1 \mathrm{~B}$ ). The dissociation constants for the binding of the intracellular domain of NCAM140 or NCAM180 to immobilized calmodulin were determined to be $\sim 8 \times 10^{-8}$ and $\sim 3 \times 10^{-8} \mathrm{M}^{-1}$, respectively (data not shown). The intracellular domains of CHL1 and L1 did not bind to calmodulin even in the presence of $1 \mathrm{~mm}$ calcium (Fig. $1 \mathrm{~B}$ ). In ELISA experiments using calmodulin as substrate coat, a concentration-dependent and saturable binding of the intracellular domains of NCAM 180 and NCAM140 was observed in the presence of $1 \mathrm{mM}$ calcium (Fig. 2 A). Using the concentration of half-maximal saturation as an approximate estimation for the dissociation constants, $\sim 7 \times 10^{-8}$ and $\sim 4 \times 10^{-8}$ were calculated as dissociation constants for the binding of the intracellular domains of NCAM180 and NCAM140 to immobilized calmodulin, respectively. These values are very similar to those determined by surface plasmon resonance analysis. This high affinity binding of the intracellular domains of NCAM to calmodulin was also observed in the presence of physiological intracellular concentrations of calcium, e.g., 100 and $250 \mathrm{~nm}$, but not in the presence of $5 \mathrm{~mm}$ EDTA (Fig. $2 \mathrm{~B}$ ).

To narrow down the binding site of calmodulin on NCAM, three peptides overlapping with the different parts of the intracellular sequence shared by NCAM140 and NCAM180 (Fig. 2C) were used in competition assays for binding of the intracellular domains of NCAM140 and NCAM180 to substrate-coated calmodulin. The peptide proximal to the transmembrane domain showed a concentration-dependent inhibition of binding of the intracellular NCAM domains to calmodulin, whereas the peptides more distal to the transmembrane domain showed no effect (Fig. 2C).

In summary, calmodulin directly binds to the membraneproximal sequences in the intracellular domain of NCAM in a calcium-dependent manner and at the physiological calcium concentrations of the cytoplasm.

\section{NCAM interacts with calmodulin in cultured hippocampal and cerebellar neurons}

Next, we addressed the question whether calmodulin and NCAM interacted in live cells. To this aim, we clustered NCAM with an antibody against its extracellular domain at the cell surface of live hippocampal neurons and analyzed by immunofluorescence staining whether calmodulin colocalized with NCAM clusters. As control, L1 or CHL1 antibodies were used for clustering. A punctate staining of clustered NCAM was seen along neurites and at the cell surface of somata (Fig. 3A). A diffuse cytoplasmic staining of calmodulin was seen in the soma of neurons, while a punctate staining was seen along neurites (Fig. 3A). A large number of NCAM-positive puncta were intensely stained by the calmodulin antibody (Fig. 3A). No coimmunostaining was observed without clustering of NCAM (Fig. $3 B$ ) or after clustering of L1 (Fig. 3C) or CHL1 (Fig. 3D). Coimmunostaining of calmodulin and clustered NCAM suggests that NCAM and calmodulin are associated with each other in neurons.

To further substantiate the interaction of NCAM with calmodulin in neurons, cultured cerebellar neurons were subjected to immunoprecipitation with NCAM antibody in the presence of calcium. Western blot analysis with calmodulin antibody showed that calmodulin was immunoprecipitated with the NCAM antibody, but not with the control nonimmune antibody (Fig. 4A), indicating that NCAM interacts with calmodulin in neurons.

\section{NCAM-induced neurite outgrowth in vitro is reduced by a calmodulin inhibitor}

To investigate whether the interaction of NCAM with calmodulin plays a role in NCAM-induced neurite outgrowth, we first analyzed cerebellar explants grown on different substrates in the absence or presence of the calmodulin inhibitor CGS9343B. Neurite outgrowth on the control substrate poly-L-lysine (PLL) was not influenced by the calmodulin inhibitor in comparison to that observed in the absence of the inhibitor, whereas the enhanced neurite outgrowth on substrate-coated NCAM was reduced to the PLL level in the presence of the calmodulin inhibitor (Fig. $4 B$ ). Quantification revealed that the NCAM-induced neurite length was reduced to $\sim 60 \%$ by the inhibitor, while neurite outgrowth on L1 and laminin was not altered in the presence of the inhibitor (Fig. 4C). The inhibitory effect of CGS9343B was also observed in primary cultures of dissociated cerebellar neurons (data not shown). Using dissociated hippocampal neurons and different calmodulin inhibitors, namely CGS9343B, W-7, and trifluoperazine, neurite outgrowth on NCAM-Fc substrate was inhibited by CGS9343B and W-7, while neurite outgrowth on PLL control substrate was not affected by CGS9343B and W-7 (Fig. 4D). Trifluoperazine was toxic to neurons. These results show that NCAM-mediated, but neither L1- nor lamininmediated neurite outgrowth depends on calmodulin.

\section{Interaction of NCAM with calmodulin is mediated by an} inverted 1-5-8-15 calmodulin binding motif in NCAM

Interestingly, the peptide deriving from the membrane-proximal sequence of the intracellular domain of NCAM that inhibits the 
A

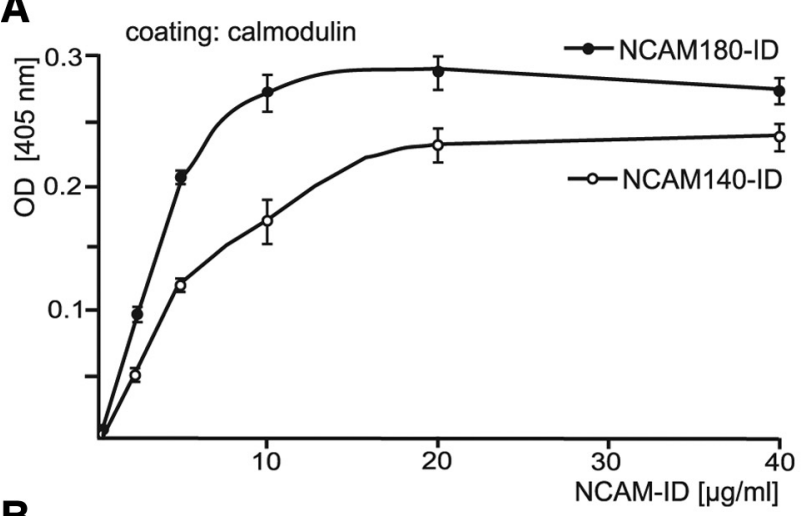

B

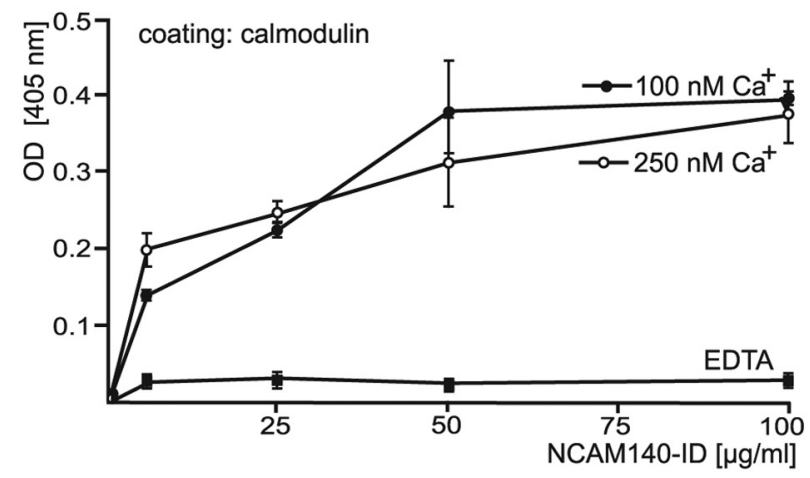

C
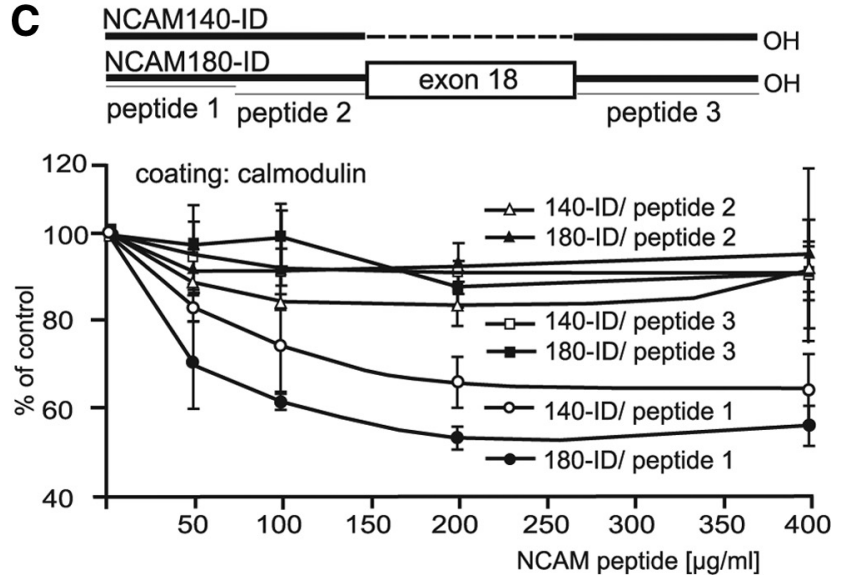

Figure 2. Calmodulin binds to the intracellular domain of NCAM140 at physiological calcium concentrations. $\boldsymbol{A}, \boldsymbol{B}$, In ELISA experiments, substrate-coated calmodulin was incubated with increasing amounts of NCAM180-ID $(\boldsymbol{A})$ and NCAM140-ID $(\boldsymbol{A}, \boldsymbol{B})$ in the presence of $1 \mathrm{mM} \mathrm{CaCl} 2$ (A) or 250 or $100 \mathrm{~nm} \mathrm{CaCl}$ or $5 \mathrm{~mm}$ EDTA (B). C, For competition ELISA, substrate-coated calmodulin was incubated with a constant amount of NCAM140-ID (open symbols) or NCAM180-ID (closed symbols) in the presence of different amounts of peptides 1 (circles), 2 (triangles), and 3 (rectangles) derived from the sequences of the intracellular domain of NCAM140 and NCAM180, which differ from NCAM140 by the presence of additional amino acids encoded by exon 18 . Binding of NCAM-IDs in the presence of the peptides is shown relative to the binding in the absence of peptides, which was set to $100 \%$. $A-C$, Binding of the NCAM-IDs was detected using the NCAM antibody 5B8. The experiments were performed three times. Error bars represent mean values \pm SD.

binding of calmodulin to the intracellular domains of NCAM contains an inverted 1-5-8-14 calmodulin binding motif (Fig. 5A; see Discussion for details). To test whether this putative calmodulin binding motif mediates the binding between NCAM and calmodulin, the sequence was mutated (Fig. 5A), and the mutated intracellular domains were analyzed by ELISA for binding to immobilized calmodulin in the presence of $1 \mathrm{~mm}$ calcium. The wild-type intracellular domains of NCAM140 and NCAM180 showed a concentration-dependent and saturable binding to substrate-coated calmodulin, whereas the mutated intracellular domains of NCAM140 and NCAM180 did not bind to substratecoated calmodulin at any concentration tested (Fig. $5 B$ ), showing that calmodulin binds to the inverted 1-5-8-14 motif within the intracellular NCAM domain.

Interaction of NCAM with calmodulin is required for NCAM-mediated neurite outgrowth of hippocampal neurons To test whether the direct binding of calmodulin to the inverted 1-5-8-14 calmodulin binding motif in NCAM is required for NCAM-induced neurite outgrowth, hippocampal neurons from NCAM-deficient mice were transfected with wild-type or mutated full-length NCAM140 and assayed for neurite outgrowth in vitro. We used NCAM140 and hippocampal neurons for this assay, since NCAM140 rather than NCAM180 or NCAM120 is sufficient for NCAM-induced neurite outgrowth of hippocampal neurons (Niethammer et al., 2002, and references therein) and since hippocampal neurons could be more efficiently transfected as compared to cerebellar neurons under conditions available to us. On PLL, neurons transfected with wild-type or mutated NCAM140 showed similar neurite lengths as neurons mocktransfected with the empty vector (Fig. 5C). Using NCAM-Fc as substrate neurite outgrowth was not affected in case of mocktransfected neurons, while it was stimulated in case of neurons transfected with wild-type NCAM140, when compared to that on PLL (Fig. 5C). Upon transfection of mutated NCAM140, neurite outgrowth was not stimulated and was comparable to that of mock-transfected neurons (Fig. $5 C$ ). These results indicate that binding of calmodulin to the 1-5-8-14 motif within NCAM is required for neurite outgrowth and are in agreement with those on neurite outgrowth in the presence of the calmodulin inhibitor (Fig. 4).

\section{Mutation of the calmodulin binding motif in NCAM alters the morphology of hippocampal neurons}

Since NCAM-mediated neurite outgrowth depends on the calmodulin binding motif in NCAM, we investigated whether disruption of this motif disturbs the trafficking of NCAM to the cell surface and thus leads to the abolishment of NCAM-mediated neurite outgrowth. Hippocampal neurons from NCAM-deficient mice were transfected with wild-type or mutated full-length NCAM140 and grown on substrate-coated NCAM-Fc. Mocktransfected NCAM-deficient neurons showed no NCAM immunostaining (data not shown). After live-cell staining with NCAM antibody, cells transfected with wild-type NCAM as well as mutated NCAM showed a punctate staining of NCAM along neurites and a punctate cell surface staining on the cell bodies of neurons (Fig. 5D), indicating that the mutated NCAM was transported to the cell surface. Interestingly, neurons expressing mutated NCAM, but not neurons expressing wild-type NCAM, showed numerous spine-like short extensions along neurites (Fig. 5E), suggesting that mutation of the calmodulin binding site leads to distinct alterations in the morphology of neurons.

Cell surface expression of NCAM is not altered by mutation of the calmodulin binding motif on NCAM

The live-cell staining of NCAM indicated that mutated NCAM was expressed at the cell surface to the same extent as wild-type NCAM. To further verify that mutation of the calmodulin binding site on NCAM did not change the extent of cell surface expression of NCAM, we performed cell surface biotinylation of 
A

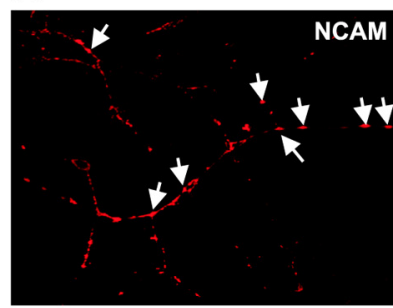

$t+t+t$

B
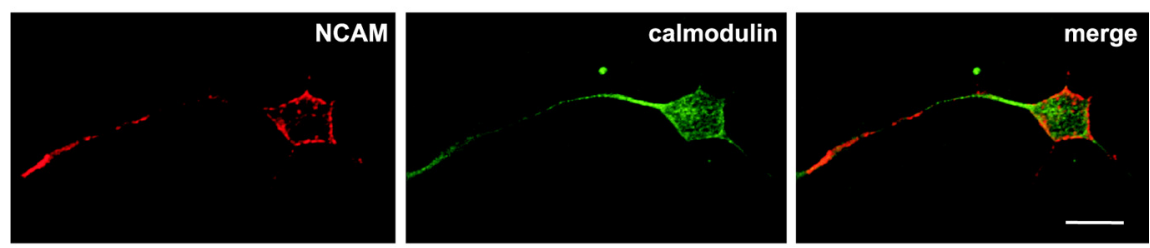

C
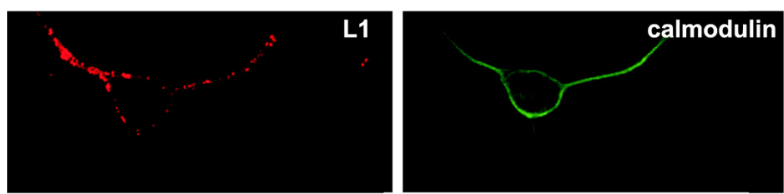

D
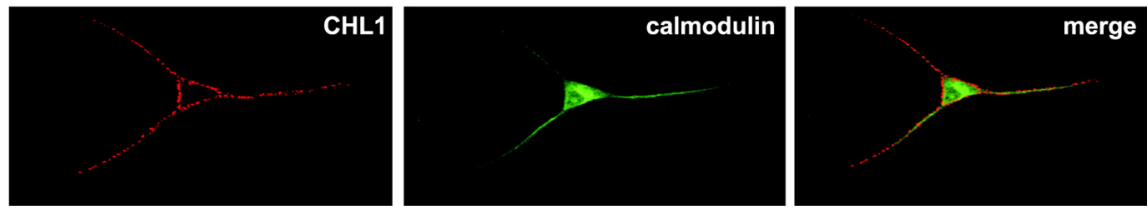

Figure 3. NCAM and calmodulin colocalize in hippocampal neurons. Live hippocampal neurons were stained with polyclona antibodies to the extracellular domains of NCAM $(\boldsymbol{A}), \mathrm{L} 1(\boldsymbol{C})$, or CHL1 (D), fixed, permeabilized, and stained with the calmodulin antibody. $\boldsymbol{B}$, Fixed hippocampal neurons were stained with NCAM and calmodulin antibodies. $\boldsymbol{A}$, Arrows indicate colocalization of NCAM and calmodulin at the cell surface of the soma and along neurites.

$\mathrm{CHO}$ cells transiently transfected with wild-type and mutated NCAM140. After biotinylation of cell surface proteins, cells were lysed and biotinylated proteins were isolated by streptavidincoupled beads and subjected to Western blot analysis using a polyclonal NCAM antibody. Both wild-type and mutated NCAM140 were detected in similar amounts as biotinylated proteins (Fig. 6A) showing that mutated and wild-type NCAM were similarly transferred to the cell surface.

Mutation of the calmodulin binding motif in NCAM does not alter palmitoylation and raft localization

of NCAM

Palmitoylation of NCAM is required for the recruitment of NCAM to lipid rafts and promotion of neurite outgrowth (Niethammer et al., 2002). Since the calmodulin binding motif overlaps with the palmitoylation site in NCAM and mutated NCAM140 was expressed at the cell surface, but did not mediate NCAM-induced neurite outgrowth, we analyzed whether palmitoylation and/or lipid raft localization of NCAM was disturbed by the mutation. To analyze the degree of palmitoylation, $\mathrm{CHO}$ cells stably transfected with wild-type and mutated NCAM140 were radiolabeled with ${ }^{3} \mathrm{H}$-palmitate. NCAM was then immunoprecipitated from the cell lysates and the precipitates were subjected to SDSPAGE and fluorography. The expression levels of NCAM in cell lysates were determined by Western blot analysis using a polyclonal NCAM antibody. This analysis revealed that the cells transfected with the mutated NCAM expressed lower amounts of NCAM when compared to cells transfected with the wildtype NCAM (Fig. 6B). Similarly, less palmitoylated NCAM was precipitated from the cells expressing mutated NCAM relative to cells expressing wild-type NCAM (Fig. 6B). Normalization of the amounts of radiolabeled, i.e., palmitoylated, NCAM to the expression levels of total NCAM revealed no reduction of the palmitoylation levels of mutated relative to the wild-type NCAM, indicating that mutation of the calmodulin binding site does not interfere with palmitoylation of NCAM.

Next, we analyzed whether the recruitment of mutated NCAM to lipid rafts was altered. We isolated lipid raft fractions and subjected these to Western blot analysis using a polyclonal NCAM antibody. Normalization of the amounts of NCAM detectable in lipid rafts to total NCAM levels showed that nearly the same amounts of total mutated and wild-type NCAM were present in lipid rafts (Fig. 6C), indicating that the lipid raft localization of NCAM is not disturbed by mutation of the calmodulin binding site.

\section{Mutation of the calmodulin binding motif in NCAM disrupts the activation} of fak but not of fyn

Several studies have shown that stimulation of NCAM leads to an activation of the fyn/fak pathway (Beggs et al., 1997; Niethammer et al., 2002; Bodrikov et al., 2005). We thus stimulated NCAMtransfected $\mathrm{CHO}$ cells using the polyclonal NCAM antibody $1 \beta 2$ directed against the extracellular domain of NCAM and analyzed whether the activation of fyn and/or fak was altered when the calmodulin binding site on NCAM was mutated. When compared to nonimmune control rabbit antibody, application of NCAM antibody to $\mathrm{CHO}$ cells transfected with the wild-type NCAM resulted in an approximately twofold increase of activated fyn (Fig. 6D). CHO cells transfected with mutated NCAM also showed an approximately twofold increase of activated fyn, while the basal level of activated fyn was reduced by $\sim 25 \%$ when compared to the basal levels observed in CHO cells transfected with wild-type NCAM (Fig. 6D). These results indicate that fyn activation is not significantly impaired by the mutation of the calmodulin binding site.

Application of the NCAM antibody to $\mathrm{CHO}$ cells transfected with the wild-type NCAM resulted in activation of fak, which is characterized by its phosphorylation, while the nonimmune control rabbit serum did not activate fak (Fig. 6E). Interestingly, activation of fak was accompanied by the generation of a phosphorylated $\mathrm{N}$-terminal $50 \mathrm{kDa}$ fragment of fak and a nonphosphorylated C-terminal $55 \mathrm{kDa}$ fragment of fak (Fig. 6E). Upon application of polyclonal NCAM antibody to CHO cells transfected with mutated NCAM, the levels of phosphorylated fulllength fak and fak fragments were reduced by $>75 \%$ relative to 
A

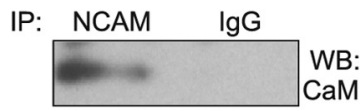

B

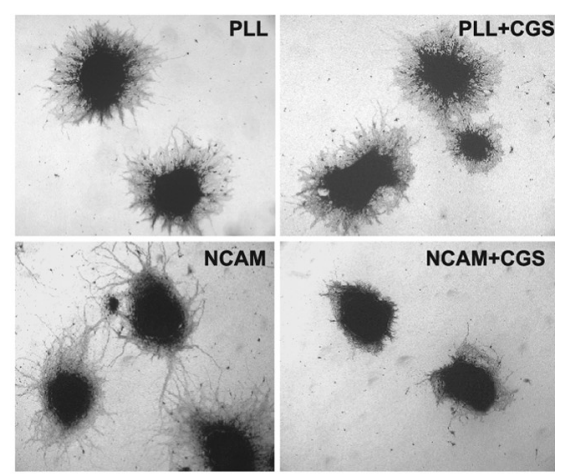

C

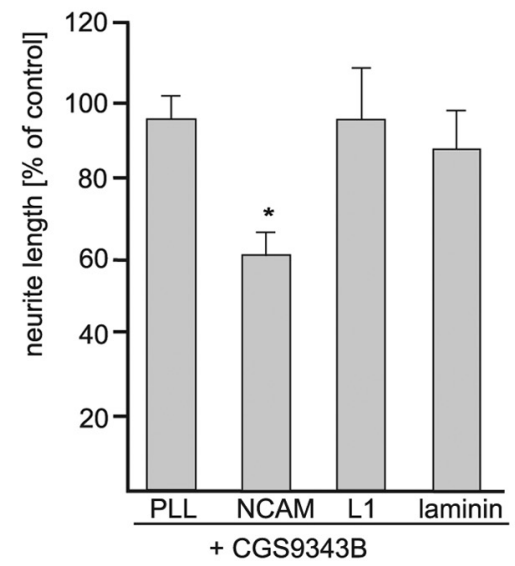

D

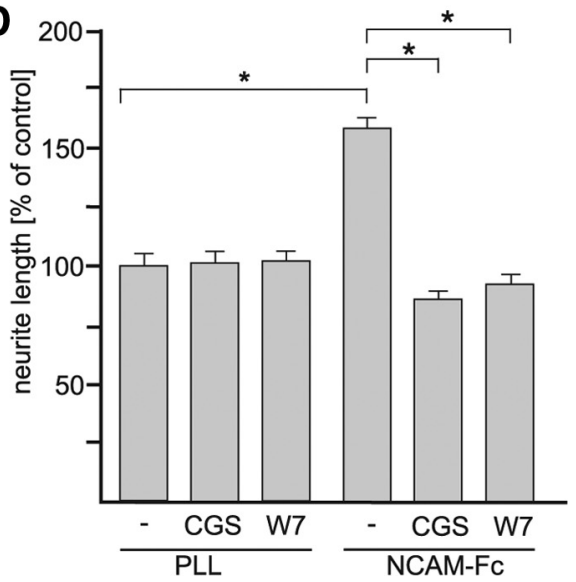

Figure 4. NCAM-induced neurite outgrowth of cultured cerebellar neurons is reduced by a calmodulin-specific inhibitor. $A$, Dissociated cerebellar neurons were subjected to immunoprecipitation with NCAM antibody $1 \beta 2$ or control nonimmune antibody (lgG) and to Western blot analysis using calmodulin (CaM) antibody. $\boldsymbol{B}$, Cerebellar microexplant cultures were incubated on substrate-coated PLL, laminin, immunopurified NCAM, or L1 in the absence or presence of the calmodulin inhibitor CGS9343B. C, Photographs of explants grown on PLL or NCAM in the absence or presence of the inhibitor are shown. Neurite outgrowth from the explants was quantified by measuring the 10 longest neurites from 10 explants. The effect of the calmodulin inhibitor on neurite outgrowth is shown relative to neurite outgrowth in the absence of the inhibitor, which was set to $100 \%$ for each substrate coat. Mean values \pm SD of three independent experiments are shown (Student's test; ${ }^{*} p<0.01$ ). D, Dissociated hippocampal neurons were grown on substrate-coated PLL or NCAM-Fc in the absence or presence of the calmodulin inhibitors CGS9343B (CGS) and W-7 (W7). Neurite lengths were measured and the effect of the calmodulin inhibitors on neurite outgrowth is shown relative to neurite outgrowth in the absence of the inhibitors, which was set to $100 \%$ for each substrate coat. Mean values \pm SEM from two independent experiments are shown (Student's $t$ test; ${ }^{*} p<0.005$ ). those observed in $\mathrm{CHO}$ cells transfected with wild-type NCAM (Fig. $6 E, G$ ). When stimulation of cells transfected with wild-type NCAM was performed in the presence of CGS9343B, phosphorylation of full-length fak and of the N-terminal fak fragment was reduced by $\sim 70 \%$ and $60 \%$ relative to that observed in the absence of the inhibitor (Fig. $6 F, G$ ), respectively.

These results indicate that the NCAM-induced activation of fak and the generation of fak fragments depend on calmodulin.

\section{NCAM stimulation leads to proteolysis of fak, resulting in transport of the generated $\mathrm{N}$-terminal fragment of fak into the nucleus}

Since it has been reported that an $\mathrm{N}$-terminal fragment of fak is imported into the nucleus (Stewart et al., 2002), we checked whether this fragment generated by NCAM stimulation enters the nucleus. $\mathrm{CHO}$ cells transfected with wild-type or mutated NCAM were subjected to subcellular fractionation to isolate nuclear and non-nuclear fractions. Western blot analysis of the subfractions showed that the $\mathrm{N}$-terminal fragment was present in both the non-nuclear and nuclear fraction upon stimulation by the polyclonal NCAM antibody, but not by a nonimmune control rabbit antibody (Fig. 7A).

Next, we investigated whether also in neurons the $\mathrm{N}$-terminal fak fragment was generated and transported into the nucleus upon NCAM stimulation. Cultured dissociated cerebellar neurons were stimulated by application of polyclonal NCAM antibody and subsequently subjected to subcellular fractionation. Upon stimulation with the NCAM antibody, the N-terminal fragment of fak and the full-length fak were present in the nonnuclear fraction (Fig. $7 B$ ), while only the N-terminal fragment (Fig. $7 B$ ), but not the $\mathrm{C}$-terminal fragment (data not shown), was detectable in the nuclear fraction. When the nonimmune control antibody was used as control for stimulation, only full-length fak was observed in the non-nuclear fraction, but not in the nuclear fraction (Fig. 7B).

These results indicate that a phosphorylated $\mathrm{N}$-terminal fragment of fak, which is generated by NCAM stimulation, is transported into the nucleus of NCAM-transfected $\mathrm{CHO}$ cells and NCAM-expressing cerebellar neurons.

\section{Stimulation of NCAM results in proteolytic generation of a C-terminal fragment of NCAM that is transported into the nucleus}

Since NCAM has been reported to be proteolytically processed (Kalus et al., 2006), we considered the possibility that a C-terminal intracellular fragment of NCAM is generated by stimulation of NCAM. Indeed, stimulation of $\mathrm{CHO}$ cells transfected with wildtype NCAM led to the generation of a C-terminal $50 \mathrm{kDa}$ fragment of NCAM as shown by Western blot analysis of cell lysates using an antibody that reacts with the C-terminal intracellular domain of NCAM (Fig. 7C). This fragment was not detected in lysates from cells transfected with mutated NCAM and when a nonimmune control rabbit antibody was used for stimulation (Fig. 7C). Western blot analysis after subcellular fractionation of $\mathrm{CHO}$ cells transfected with wild-type NCAM140 or nontransfected cerebellar neurons showed that the C-terminal NCAM fragment was detected in the non-nuclear, but also in the nuclear, fraction upon stimulation with NCAM antibody, but not with nonimmune control rabbit antibody (Fig. $7 D, E$ ). Surprisingly, the $50 \mathrm{kDa}$ fragment of NCAM in the nuclear fraction of cerebellar neurons was recognized by an antibody against the extracellular domain of NCAM (Fig. 7E). 
A

WTNCAM: MDITCYFLNKCGLIMCIAVNLCGKAGPGAKGKDMEEG mutNCAM: MDITCYYMNKCGMLMCIAVNMCGKAGPGAKGKDMEEG

\section{B}

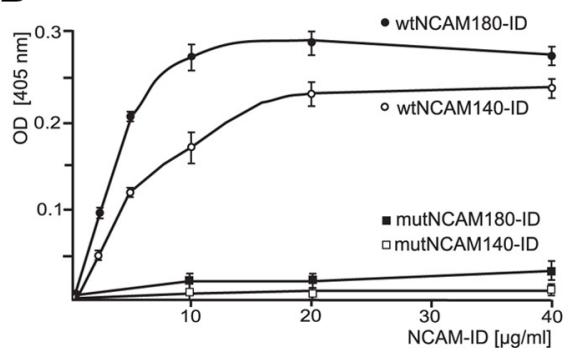

D

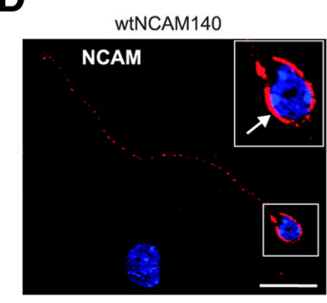

C

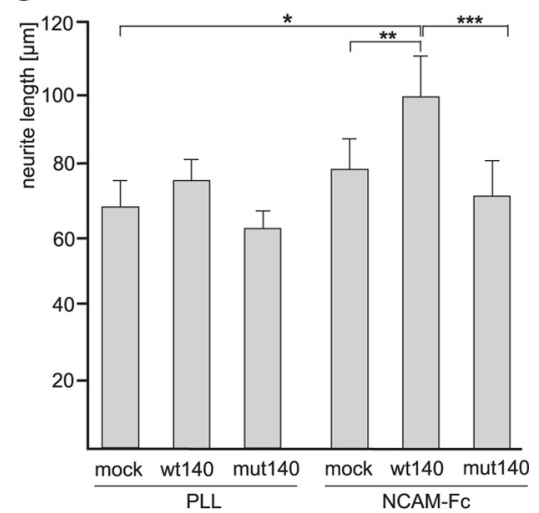

E

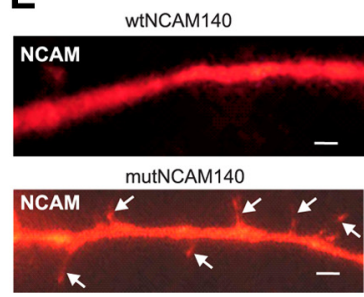

Figure 5. Characterization of the calmodulin binding site on NCAM. $\boldsymbol{A}$, The depicted sequence stretch "wtNCAM" matches the sequence of the synthetic NCAM peptide 1, which inhibits binding of NCAM-IDs to calmodulin (see Fig. 2C) and contains the inverted 1-5-8-14 calmodulin binding motif (the hydrophobic amino acids in position 1, 5, 8, and 14 are indicated) and the palmitoylation sites (underlined cysteine residues). The sequence "mutNCAM" shows the sequence of mutated NCAM-ID (amino acid exchanges are indicated in bold) obtained after alteration of the calmodulin binding motif. $\boldsymbol{B}$, In ELISA experiments, substratecoated calmodulin was incubated with increasing amounts of wild-type NCAM140-ID (wtNCAM140-ID) and NCAM180-ID (wtNCAM180-ID) and mutated NCAM180 (mutNCAM180-ID) and NCAM140 (mutNCAM140-ID). Binding of the NCAM-IDs was detected by using the NCAM antibody 5B8. The experiments were performed at least three times. Error bars represent mean values \pm SD. C, Live hippocampal neurons from NCAM-deficient mice were cotransfected with GFP-expressing vector and an empty plasmid (mock) or with a plasmid coding for full-length wild-type NCAM140 (wt140) or mutated NCAM140 (mut140). Neurons were grown on substrate-coated PLL or NCAM-Fc. Neurite lengths of GFP-positive neurons were determined. Mean values $\pm S D$ from three independent experiments are shown (Student's $t$ test; ${ }^{* * *} p<0.005,{ }^{* *} p<0.01,{ }^{*} p<0.05$ ). D, E, Hippocampal neurons from NCAM-deficient mice transfected with wild-type NCAM140 (wtNCAM140) or mutated NCAM140 (mutNCAM140) and grown on substrate-coated NCAM-Fc were incubated with NCAM antibody $1 \beta 2$. After fixation, secondary antibody and DRAQ5 were applied. Neurons with surface expression of NCAM (red) and staining of nuclei (blue) are shown. Arrows in the insets indicate cell surface staining of NCAM. Scale bars represent $20 \mu \mathrm{m} . \boldsymbol{D}, \boldsymbol{E}$, Arrows indicate aberrant spine-like structures seen along neurites of neurons that express mutated NCAM and are grown on substrate-coated NCAM-Fc. $\boldsymbol{E}$, Neurites are shown at high magnification. Scale bars represent $1 \mu \mathrm{m}$.

To further verify that the nuclear $50 \mathrm{kDa}$ fragment, which is recognized by the antibody against the extracellular domain of NCAM, is NCAM specific, we isolated nuclei from brains of wildtype and NCAM-deficient mice and subjected them to Western blot analysis with NCAM antibody against the extracellular domain. The $50 \mathrm{kDa}$ NCAM fragment was detected in nuclei from wild-type brains, but not in nuclei from NCAM-deficient brains (Fig. $7 F$ ), supporting that the $50 \mathrm{kDa}$ fragment is a NCAM fragment.

In a next step, we elucidated whether stimulation of cerebellar neurons by NCAM-Fc also triggered the transport of this unusual $50 \mathrm{kDa}$ NCAM fragment to the nucleus, and found the 50 $\mathrm{kDa}$ fragment in the nuclear fraction after stimulation with NCAM$\mathrm{Fc}$, but not after treatment with $\mathrm{Fc}$ alone (Fig. $7 G$ ). In comparison to the stimulation with NCAM antibody, the amounts of NCAM fragment in the nuclear fraction were smaller upon stimulation with NCAM-Fc (Fig. 7G), suggesting that the clustering of NCAM by antibody is a stronger signal for triggering nuclear import of the NCAM fragment than the triggering homophilic NCAM interaction by NCAM-Fc.

Since it appeared likely that the NCAM fragment is imported into the nucleus in association with calmodulin, we determined the amounts of calmodulin in the nucleus after stimulation of $\mathrm{CHO}$ cells and cerebellar neurons with NCAM or, as control, with nonimmune antibodies. Interestingly, a considerable amount of calmodulin was found in the nuclear fraction under control condition, i.e., after incubation with the nonimmune antibody. An approximately twofold increase of the amount of calmodulin in the nuclear fraction relative to control conditions was observed after NCAM stimulation (Fig. 7H). This result indicates that nuclear import of the NCAM fragment, which is triggered by NCAM stimulation, is accompanied by a concomitant nuclear import of calmodulin, probably by association with the NCAM fragment during the translocation into the nucleus.

To investigate whether the $50 \mathrm{kDa}$ NCAM fragment was generated by proteolytic cleavage and to identify the responsible protease(s), NCAM stimulation and subcellular fractionation was performed in the presence of protease inhibitors. The generation of the fragment and its transport was not altered in the presence of the $\gamma$-secretase inhibitor DAPT (Fig. 7I) or the metalloprotease inhibitor GM6001 (data not shown). On the one hand, this observation indicates that the $50 \mathrm{kDa}$ fragment was not generated by a process called RIP (regulated intramembrane proteolysis), which involves metalloproteases of the ADAM family, like the NCAM-cleaving ADAM17/TACE (Kalus et al., 2006) and $\gamma$-secretase, and leads to the generation of intracellular fragments devoid of any extracellular portions. On the other hand, this observation suggests that the fragment is derived from cleavage by an extracellularly active protease. The prime candidate for this cleavage is plasmin, as it has been reported that NCAM is cleaved by plasmin (Endo et al., 1998; Endo et al., 1999). To test this possibility, CHO cells transfected with NCAM were stimulated with NCAM antibody in the presence of the serine protease inhibitor aprotinin, which efficiently inhibits plasmin and which also has been shown to inhibit the plasmin cleavage of NCAM (Endo et al., 1998, 1999). Aprotinin reduced the generation of the NCAM fragment by $30-50 \%$ and completely abolished the transport of the fragment into the nucleus (Fig. 7I).

If the C-terminal NCAM fragment consisted of intracellular and extracellular parts and was generated from the cleavage by an extracellular protease in the extracellular domain of NCAM, a complementary soluble fragment comprising the extracellular domain of NCAM had to be released into the cell culture supernatant upon NCAM stimulation. To test this idea, cell culture supernatants of $\mathrm{CHO}$ cells transfected with wild-type NCAM were analyzed after application of nonimmune control or NCAM antibody. As previously shown (Kalus et al., 2006), a soluble 110 $\mathrm{kDa}$ NCAM fragment resulting from the cleavage by ADAM17/ TACE was detected in the culture supernatant when cells were 
A

\begin{tabular}{ll}
\multicolumn{2}{c}{ lysate } \\
wt mut
\end{tabular}

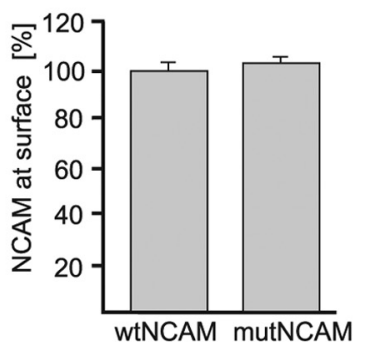

D
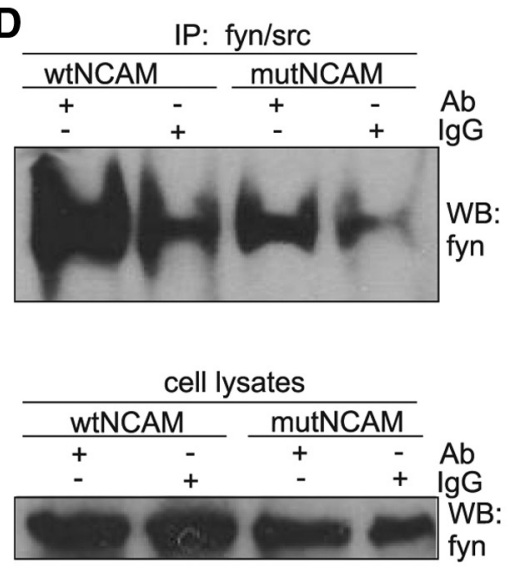

B
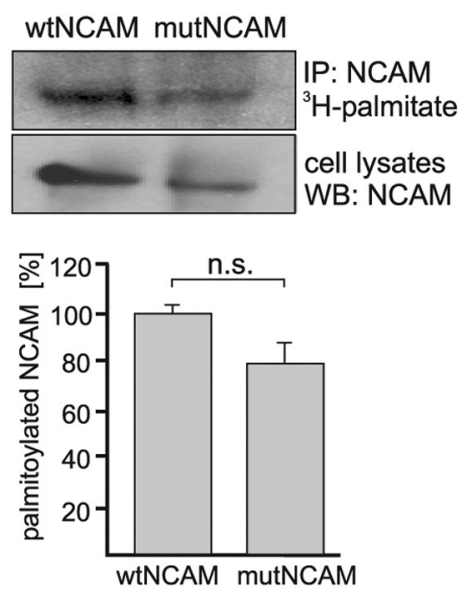

E
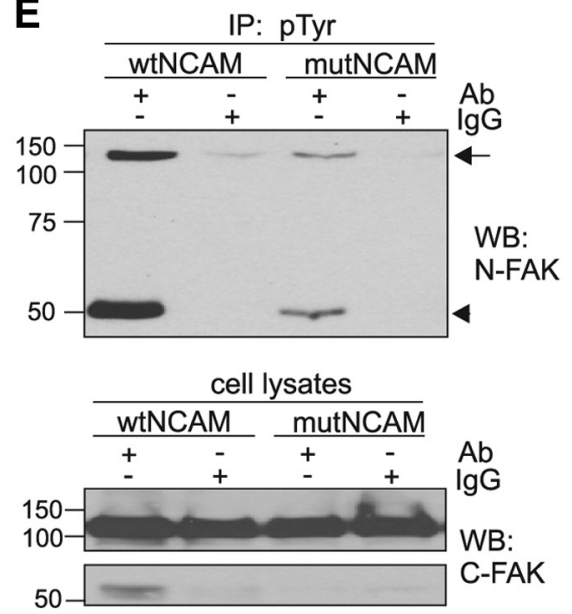

C wtNCAM mutNCAM rafts lysate rafts lysate
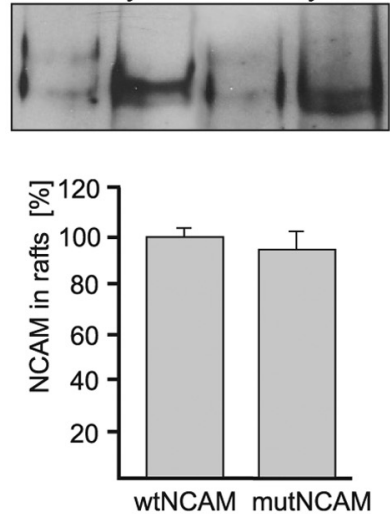

$\mathbf{F}$

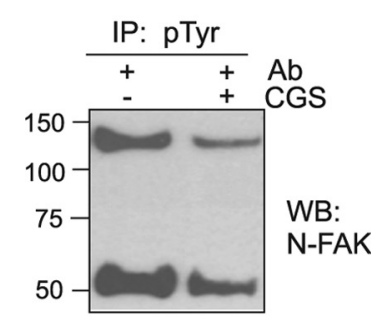

G

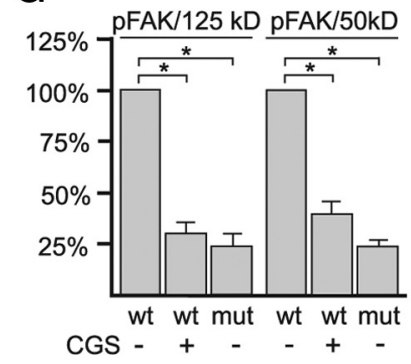

Figure 6. Effect of mutating the calmodulin binding motif in NCAM on NCAM subcellular localization and activation of the fyn/fak pathway. $A-C, C H O$ cells transiently $(\boldsymbol{A})$ or stably $(B, C)$ transfected with wild-type NCAM140 (wt) or mutated NCAM140 (mut) were subjected to cell surface biotinylation $(\boldsymbol{A})$, labeled with radioactive palmitate, and subjected to immunoprecipitation with polyclonal NCAM antibody $(\boldsymbol{B})$ or to lipid raft isolation ( $\boldsymbol{C}$. Cell lysates $(\boldsymbol{A}-\boldsymbol{C}$, biotinylated surface proteins isolated by streptavidin-coupled beads (surface) $(\boldsymbol{A})$, and lipid raft fractions $(\boldsymbol{C})$ were subjected to Western blot analysis using NCAM antibody 5B8. $\boldsymbol{B}$, Immunoprecipitates from radiolabeled cells were analyzed by SDS-PAGE and fluorography. Quantifications of the amount of biotinylated NCAM (A), of radioactively labeled NCAM ( $\boldsymbol{B})$, and of NCAM in the lipid raft fraction $(\boldsymbol{C}$ relative to the total amount of NCAM from three independent experiments are shown in the lower panels (n.s. $=$ not significant; Student's $t$ test). $A-C$, The level of wild-type NCAM was set to $100 \%$. D, E, CHO cells transiently transfected with wild-type NCAM140 (wt) or mutated NCAM140 (mut) were incubated with control nonimmune antibody (lgG) or NCAM antibody $1 \beta 2$ (Ab) and subjected to immunoprecipitation and Western blot analyses. D, Activated fyn dephosphorylated at Tyr-531 was immunoprecipitated using an antibody against src dephosphorylated at Tyr-527 and cross-reacting with fyn dephosphorylated at Tyr-531. Activated fyn (upper panel) and total fyn in the lysates (lower panel) were detected by Western blot analysis using a fyn-specific antibody. $\boldsymbol{E}$, Tyrphosphorylated activated fak was immunoprecipitated using an antibody against phospho-tyrosine (pTyr) and subjected to Western blot analysis using fak-specific antibodies. Full-length fak (arrow) and N-terminal fak fragment (arrowhead) are detected in the immunoprecipitates using an antibody against the N-terminal part of fak (WB: N-FAK; upper panel). Total fak (middle panel, short exposure) and C-terminal fak fragment (lower panel, long exposure) are seen in the lysates with an antibody against the C-terminal part of fak (WB: C-FAK).F, CHO cells transiently transfected with wild-type NCAM140 were stimulated with NCAM antibody $1 \beta 2$ in the absence or presence of the calmodulin inhibitor CGS9343B and subjected to immunoprecipitation. Phosphorylated full-length fak and N-terminal fak fragment are detected by Western blot analysis of the pTyr antibody immunoprecipitates using an antibody against the N-terminal part of fak (WB: N-FAK). G, Determination of the relative levels of phosphorylated full-length fak (pFAK/125 kDa) and fak fragment (pFAK/50 KDa) is shown. The levels after stimulation of cells expressing mutated NCAM (mut) or after treatment of cells expressing wild-type NCAM (wt) with CGS9343B are related to the levels obtained after stimulation of cells expressing wild-type NCAM (wt), which was set to $100 \% . A-C, G$, Quantifications as mean values \pm SD of three independent experiments are shown.

incubated with the nonimmune control antibody, signifying basal levels of NCAM proteolysis. After stimulation with NCAM antibody, the level of this fragment was reduced, while a soluble $55 \mathrm{kDa}$ fragment appeared (Fig. 7J). This result suggests that NCAM stimulation interferes with the generation of the $110 \mathrm{kDa}$ NCAM fragment in favor of the cleavage of NCAM by a serine protease, most likely plasmin, and leads to generation of an extracellular soluble N-terminal $55 \mathrm{kDa}$ fragment and a $50 \mathrm{kDa}$ $\mathrm{C}$-terminal fragment consisting of intracellular and extracellular domains.
NCAM and fak colocalize in the nuclei of cerebellar neurons after NCAM stimulation

To confirm the nuclear localization of NCAM upon NCAM stimulation, cultures of dissociated cerebellar neurons were incubated with polyclonal NCAM antibody or nonimmune control antibody. Neurons were subjected to immunofluorescence staining of NCAM using a monoclonal NCAM antibody against the intracellular domain of NCAM and a nuclear marker. With the control antibody, no NCAM immunoreactivity was detectable in the nucleus (data not shown). Upon stimulation with NCAM 

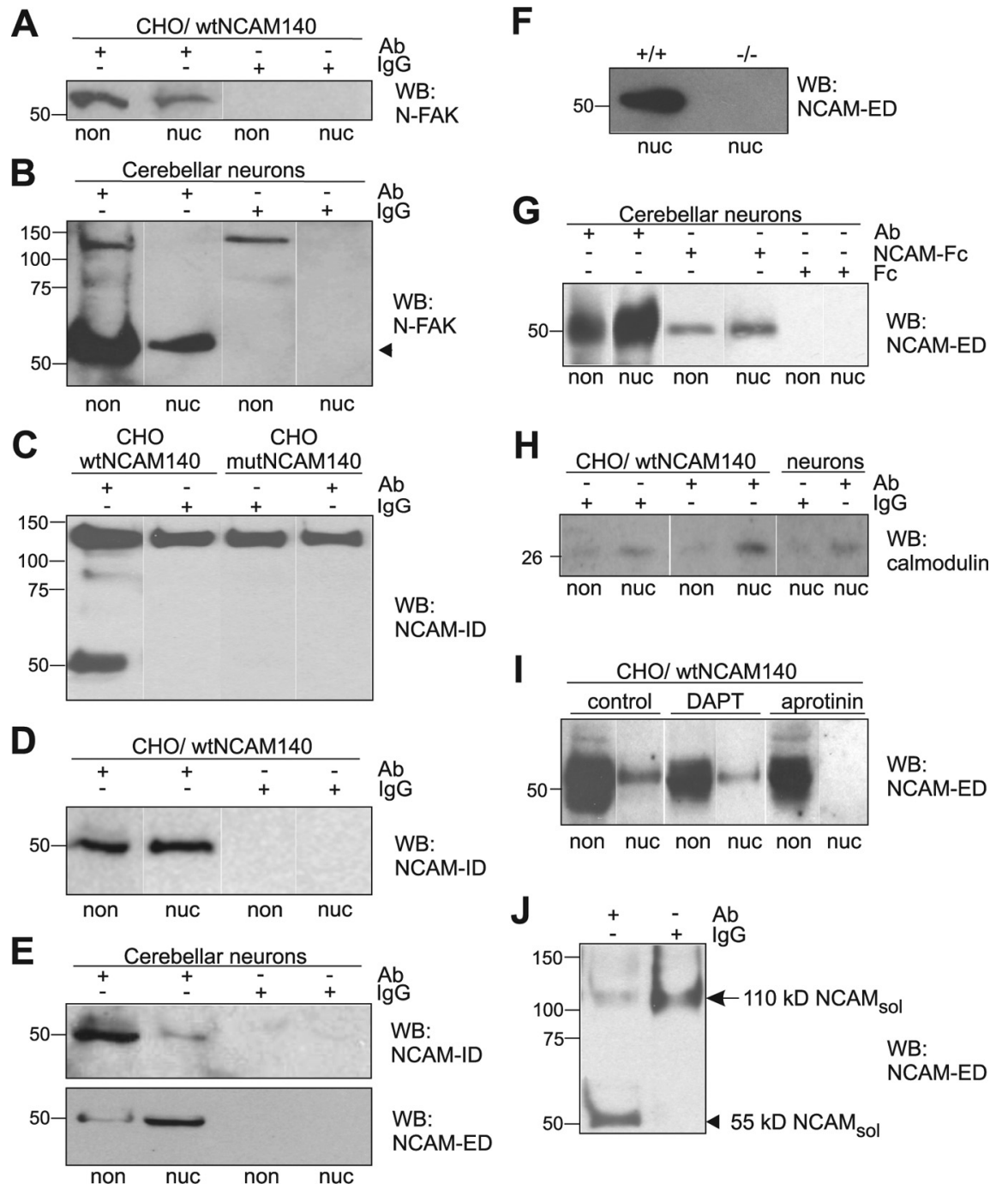

Figure 7. Generation and nuclear import of fak and NCAM fragments after NCAM stimulation. CHO cells transiently transfected with wild-type NCAM140 (wt) or mutated NCAM140 (mut) $(\boldsymbol{A}, \boldsymbol{C}, \boldsymbol{D}, \boldsymbol{H}-\boldsymbol{J})$ or cerebellar neurons $(\boldsymbol{B}, \boldsymbol{E}, \boldsymbol{G})$ were incubated with control nonimmune antibody $(\mathrm{Ig})(\boldsymbol{A}-\boldsymbol{E}, \boldsymbol{H}, \boldsymbol{J})$ and NCAM antibody $1 \beta 2(\mathrm{Ab})(\boldsymbol{A}-\boldsymbol{E}, \boldsymbol{G}-\boldsymbol{J})$ in the absence $(\boldsymbol{A}-\boldsymbol{E}, \boldsymbol{G}-\boldsymbol{J})$ or presence of aprotinin or DAPT $(\boldsymbol{I})$ or NCAM-Fc or Fc $(\boldsymbol{G})$. C, Lysates of transfected $\mathrm{CH} 0$ cells were subjected to Western blot analysis. $\boldsymbol{F}$, Nuclei were isolated from brains of wild-type (+/+) or NCAM-deficient (-/-) mice. $\boldsymbol{A}, \boldsymbol{B}, \boldsymbol{D}, \boldsymbol{E}, \boldsymbol{G}, \boldsymbol{H}, \boldsymbol{I}$, The transfected CHO cells or neurons were subjected to subcellular fractionation resulting in a non-nuclear (non) and a nuclear (nuc) fraction. The subfractions were subjected to Western blot analysis using an antibody against the N-terminal part of fak (WB: N-FAK) $(\boldsymbol{A}, \boldsymbol{B})$, the NCAM antibody P61 recognizing the intracellular domains of NCAM140 and NCAM180 (WB: NCAM-ID) (C-E), the NCAM antibody $1 \beta 2$ against the extracellular domain of NCAM (WB: NCAM-ED) $(\boldsymbol{E}-\boldsymbol{G}, \boldsymbol{I})$, and calmodulin $(\boldsymbol{H}) . \boldsymbol{J}$, After stimulation, cell culture supernatants were collected and subjected the Western blot analysis using the monoclonal antibody $\mathrm{H} 28$ against the extracellular domain of NCAM (WB: NCAM-ED). The soluble NCAM fragments are indicated. $A-J$, A representative result from three independent experiments is shown.

antibody, NCAM immunoreactivity was detectable in the nucleus (Fig. 8). Scanning through the different layers of the cultured neurons by laser confocal microcopy showed NCAM staining along neurites and at the surface of the cell body in a lower substrate-proximal layer (Fig. $8 \mathrm{~A}$ ). In a medial layer, no NCAM staining along neurites and on the cell body was observed. However, in this layer, NCAM staining overlapped with the staining of a nuclear marker, indicating that NCAM was present in the nucleus (Fig. $8 A, C$ ). In a similar experiment, we analyzed whether NCAM colocalized with fak in the nucleus and indeed found colocalization of NCAM and fak in the nucleus using a mouse antibody against the intracellular domain of NCAM and a rabbit antibody against the $\mathrm{N}$-terminal fak (Fig. $8 B, D$ ). To test the possibility that
NCAM also colocalizes with calmodulin in the nucleus, a rabbit antibody against the extracellular domain of NCAM and a mouse antibody against calmodulin were used. NCAM and calmodulin immunoreactivities were observed in the nucleus, but no significant colocalization of NCAM and calmodulin was detectable (Fig. $8 E$ ). The immunolocalization of NCAM by antibodies against its intracellular and the extracellular domains and of fak in the nucleus indicates that NCAM stimulation triggers the nuclear transport of a transmembrane NCAM fragment with an extracellular stub and of a fak fragment.

\section{Trafficking of the NCAM fragment via the ER to the nucleus is calmodulin dependent}

The detection of a NCAM fragment that contains not only the intracellular domain but also the transmembrane domain and part of the extracellular domain raised the question of how this fragment is transported to the nucleus. In the last decade, a number of studies showed that even fulllength cell surface receptors such as CD44 (Lee et al., 2009), dystroglycan (Oppizzi et al., 2008), or ErbB-2, Erb-B3, and ErbB-4 as well as the EGF, FGF, angiotensin, endothelin, or bradykinin receptors (for references and review, see Planque, 2006) are translocated to the nucleus after internalization. It has been reported that the EGF receptor is translocated to the ER, where it associates with the sec61 translocon (Liao and Carpenter, 2007). The transmembrane EGF receptor is then extracted from the ER membrane and translocated to the cytoplasm, from where it is imported to the nucleus in a hsp70- and importindependent manner.

To test whether the transmembrane NCAM fragment is transported to the nucleus via a similar pathway, we subjected Neuro2a cells to NCAM stimulation after cell surface biotinylation and analyzed subcellular fractions for the presence of biotinylated NCAM fragment. The Neuro2a cell line was chosen for this experiment because Neuro2a cells express NCAM140 (Kalus et al., 2006), are available in large quantities, and showed generation and nuclear import of the $50 \mathrm{kDa}$ NCAM fragment after stimulation with NCAM antibody (Fig. 9A), but not after incubation with a nonimmune control antibody (Fig. 9A) or an L1 antibody, which also reacts with Neuro2a cells (data not shown). After NCAM stimulation and isolating biotinylated proteins using streptavidin beads, Western blot analysis with NCAM antibody revealed that the biotinylated $50 \mathrm{kDa}$ NCAM fragment was present in both the smooth ER (SER) and rough ER (RER) fractions (Fig. 9B), which both were enriched in the ER marker protein calreticulin (Fig. 9C). Moreover, the biotinylated $50 \mathrm{kDa}$ NCAM fragment was also detectable in the cytoplasmic fraction 
and nuclear fractions (Fig. 9B), which were enriched in the cytoplasmic marker protein actin and the nuclear marker protein HP1 (Fig. 9C), respectively. The biotinylated NCAM fragment was not detectable after incubation with a control nonimmune antibody (Fig. 9B) or an L1 antibody (data not shown). These results indicate that the transmembrane NCAM fragment is translocated to the ER and released into the cytoplasm after internalization, which is triggered by NCAM stimulation.

To test whether the NCAM fragment was released from the ER and whether it was translocated from the cytoplasm to the nucleus, the ER fraction containing the biotinylated NCAM fragment was incubated with cytoplasm from untreated Neuro2a cells. For the nuclear import, the cytoplasmic fraction with the biotinylated NCAM fragment was incubated with nuclei from untreated Neuro2a cells. No biotinylated NCAM fragment was released from the ER membranes into the cytoplasm (Fig. 9D), and no import of biotinylated NCAM fragment into the nucleus (data not shown) was observed when fractions that had been isolated in the presence of EGTA were used.

It has been reported that calmodulin functions as a carrier protein for nuclear transport and mediates a nonconventional nuclear import that is GTP-, importin-, and ran-independent but is regulated by intracellular calcium (for review, see Wagstaff and Jans, 2009). Although this nonconventional calmodulinand calcium-dependent nuclear import has been reported only for soluble proteins, but not for transmembrane proteins, we tested the possibility that the import of the NCAM fragment into the nucleus and the putative release of the NCAM fragment from the ER membrane is mediated by calmodulin in a calciumdependent manner. Therefore, ER, cytoplasmic, and nuclear fractions were isolated in the absence of EGTA, which depletes the level of free calcium and was used in the experiments where no translocation from the ER to the cytoplasm and no nuclear import were observed. After incubating the SER fraction containing biotinylated NCAM fragment with cytoplasm from nonstimulated untreated cells in the presence of calcium alone or together with CGS9343B, the biotinylated NCAM fragment was released from the ER membrane in the presence of calcium, but not in the presence of the inhibitor (Fig. 9E). The biotinylated NCAM fragment was imported into nuclei after incubating cytoplasm containing biotinylated NCAM fragment with nuclei from nonstimulated untreated cells in the presence of calcium, but not in the presence of CGS9343B (Fig. 9F). Incubation of SER with purified calmodulin in the presence of calcium, but not in the absence of calcium, resulted in the release of biotinylated NCAM fragment (Fig. 9G). However, no nuclear import of NCAM fragment was detectable when nuclei were incubated with purified calmodulin (data not shown). These results indicate that the translocation of the NCAM fragment from the ER membrane to the cytoplasm and the import of the NCAM fragment from the cytoplasm into the nucleus are mediated by calmodulin in a calcium-dependent manner.

Since the import of NCAM into the nucleus depends on calmodulin, we investigated whether biotinylated NCAM in the cytoplasmic fraction is associated with calmodulin. After immunoprecipitation of calmodulin and isolation of biotinylated proteins by streptavidin beads, Western blot analysis using an antibody against the extracellular domain of NCAM revealed that biotinylated NCAM fragment was coprecipitated with the calmodulin antibody, but not with a nonimmune control antibody (Fig. 9H). This result shows that transmembrane NCAM binds to calmodulin in the cytoplasm after or during translocation.

To investigate whether the NCAM fragment is also present in ER fractions from mouse brain and whether it is released from the ER membrane and imported into the nuclei, we isolated ER fractions and cytoplasm from mouse brain. The cytoplasmic fraction was then incubated either with nuclear or ER fraction. Western blot analysis showed that the NCAM fragment was present in the ER fraction, was released from the ER membranes after incubation with cytoplasmic fraction (Fig. 9I), and was imported into nuclei after incubation with the cytoplasmic fraction (Fig. 9J). The NCAM fragment was not released from the ER membranes nor imported into the nuclei when the homogenization buffer for 
A

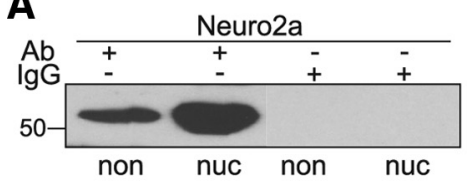

D

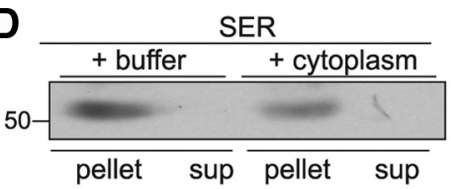

H

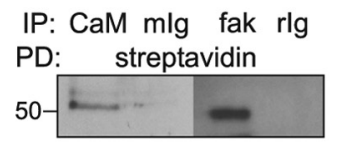

B

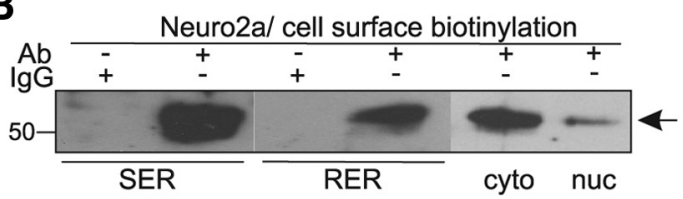

E

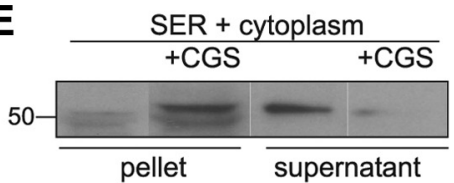

I

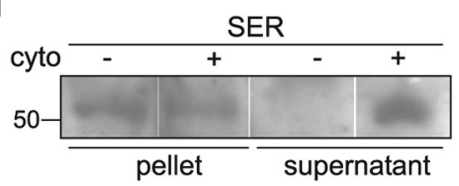

$F$
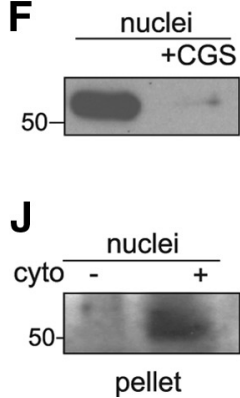

C
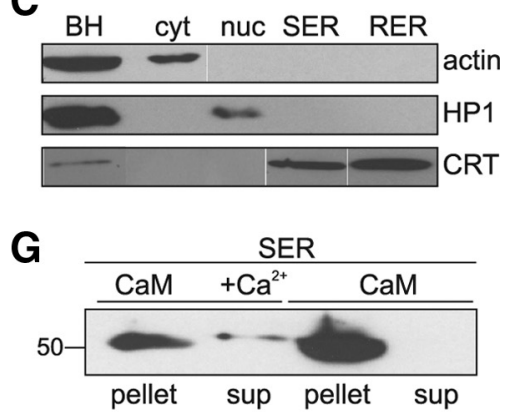

K

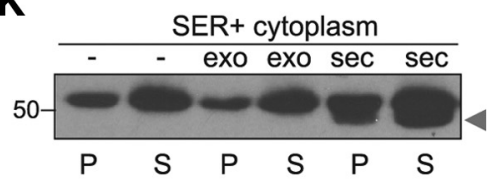

Figure 9. Trafficking of the transmembrane NCAM fragment from the cell surface via the ER and the cytoplasm to the nucleus. $\boldsymbol{A}$, Neuro2a cells were incubated with NCAM (Ab) or with control nonimmune antibody ( $\mathrm{gG}$ ) and subjected to subcellular fractionation resulting in a non-nuclear (non) and a nuclear (nuc) fraction. The subfractions were subjected to Western blot analysis using the NCAM antibody $1 \beta 2$ against the extracellular domain of NCAM. $\boldsymbol{B}-\boldsymbol{H}$, Neuro2a cells were subjected to cell surface biotinylation, NCAM stimulation, and subcellular fractionation. $\boldsymbol{B}$, Using streptavidin-coupled beads, biotinylated proteins were isolated from the SER, RER, cytoplasmic (cyto), and nuclear (nuc) fractions and the biotinylated NCAM fragment (arrow) was detected by Western blot analysis using the NCAM antibody $1 \beta 2$. C, SER, RER, cytoplasmic (cyt), and nuclear (nuc) fractions were probed by Western blot analysis with actin, HP1, and calreticulin (CRT). D, A SER fraction containing biotinylated NCAM fragment were isolated in the presence of EDTA and incubated with the cytoplasm that was isolated from untreated cells in the presence of EDTA or was incubated with EDTA-containing buffer used for the isolation of SER and cytoplasm. $\boldsymbol{E}-\boldsymbol{G}, \operatorname{SER}(\boldsymbol{E}, \boldsymbol{G})$ or cytoplasmic $(\boldsymbol{F})$ fractions containing biotinylated NCAM fragment were isolated in the presence of calcium and incubated with the cytoplasm $(\boldsymbol{E})$ and nuclei $(\boldsymbol{F})$ of untreated cells or with recombinant calmodulin $(\boldsymbol{C a M})(\boldsymbol{G})$ in the presence of calcium $(\boldsymbol{E}-\boldsymbol{G})$ and/or the calmodulin inhibitor CGS9343B (+CGS) (E, F). (D-G) After centrifugation at 100,000 $\times g(\boldsymbol{D}, \boldsymbol{E}, \boldsymbol{G})$ or $1000 \times g(\boldsymbol{F})$, biotinylated proteins were isolated from the pellet $(\boldsymbol{D}-\boldsymbol{G})$ and supernatant (sup) $(\boldsymbol{D}, \boldsymbol{E}, \boldsymbol{G})$ fractions and the biotinylated NCAM fragment (arrow) was detected by Western blot analysis using the antibody $1 \beta 2$. $\boldsymbol{H}$, The cytoplasmic fraction containing the biotinylated NCAM fragment was subjected to immunoprecipitation using a mouse calmodulin antibody (CaM) or rabbit antibody against the N-terminal part of fak (fak) or nonimmune mouse (mlg) or rabbit (rlg) antibodies followed by pull-down of biotinylated proteins from the immunoprecipitates. The biotinylated NCAM fragment (arrow) was detected by Western blot analysis using the antibody $1 \beta 2$. I-K, SER, cytoplasm, and nuclei were isolated from mouse brain and SER $(\boldsymbol{I}, \boldsymbol{K})$ or nuclei $(\boldsymbol{J})$ were incubated with the cytoplasmic fraction (cyto) in the absence $(\boldsymbol{I}-\boldsymbol{K})$ or presence of exotoxin $A($ exo) or sec61 $\beta$ antibody $($ sec) $(\boldsymbol{K})$. After centrifugation at $100,000 \times g(\boldsymbol{I}, \boldsymbol{K})$ or $1000 \times g(\boldsymbol{J})$, the pellets $(\boldsymbol{I}-\boldsymbol{K})$ and supernatants $(\boldsymbol{I}, \boldsymbol{K})$ were subjected to Western blot analysis using the NCAM antibodies $1 \beta 2(\boldsymbol{I}, \boldsymbol{J})$ or P61 $(\boldsymbol{K})$.

$\boldsymbol{A}-\boldsymbol{K}$, A representative result from three independent experiments is shown.

isolation of the cytoplasmic fraction was used (Fig. 9I). These results indicate that in the brain the transport of the transmembrane NCAM fragment to the nucleus follows the same pathway as observed for Neuro2a cells.

To test whether the NCAM fragment was released from the ER in dependence of the sec61 translocon, the ER fraction from brain was incubated with brain cytoplasm in the presence of calcium and in the absence or presence of a sec $61 \beta$ antibody or exotoxin A, which both block retrotranslocation of EGF receptor (Liao and Carpenter, 2007). The release of the NCAM fragment from the ER membranes into the cytoplasm was not affected neither by sec61 $\beta$ antibody nor by exotoxin A (Fig. 9K), indicating that the NCAM fragment is not retrotranslocated by a sec61 translocondependent mechanism as reported for the EGF receptor.

\section{Discussion}

\section{Binding of calmodulin to NCAM is mediated by an inverted} 1-5-8-14 calmodulin binding motif

In the present study, we show that calmodulin directly binds to the intracellular domain of NCAM140 and NCAM180 with high affinity in a calcium-dependent manner, while it does not bind to the intracellular domains of two other closely related cell adhesion molecules of the Ig superfamily, L1 and CHL1, which do not contain this motif. In previous studies, so-called 1-5-10, 1-8-14, or 1-5-8-14 motifs have been identified as calcium-dependent calmodulin binding motifs. These motifs are designated 1-5-10, 1-5-8-14, and 1-8-14 according to the positions of conserved hydrophobic amino acid residues within the consensus sequence

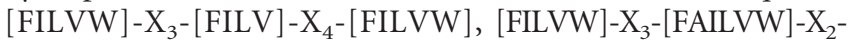
[FAILVW]-X ${ }_{5}-\left[\right.$ FILVW], or $\left[\right.$ FILVW]-X ${ }_{6}-\left[\right.$ FAILVW]-X ${ }_{5}-[$ FILVW] and have a net charge of ranging from -1 to +6 , respectively (Rhoads and Friedberg, 1997). Due to the pseudo-twofold symmetry of the two lobes of calmodulin it is possible that the motifs can also bind to calmodulin in a reverse orientation. Here, we identified a potential inverted 1-5-8-14 motif with a net charge of +1 and an overlapping 1-5-10 motif with a net charge of 0 near the transmembrane domain in the intracellular domains of NCAM140 and NCAM180 of human, bovine, rat, mouse, chicken, Xenopus, newt, and zebrafish. A peptide covering these motifs inhibited the binding of the intracellular domains of NCAM to calmodulin, showing that binding of NCAM to calmodulin is mediated by this calmodulin binding motif. Disruption of this motif by mutation completely abolished binding of calmodulin to the intracellular domain of NCAM.

\section{Interaction of calmodulin with NCAM is essential for NCAM-mediated neurite outgrowth and influences neuronal morphology}

In the presence of the calmodulin inhibitor CGS9343B, which specifically binds to calmodulin and inhibits the interaction with its binding partners (Norman et al., 1987), NCAM-dependent neurite outgrowth of cerebellar and hippocampal neurons was reduced, while the laminin- and L1-dependent neurite outgrowth was unaffected. Furthermore, disturbance of the direct interaction of calmodulin with NCAM by mutating the calmodulin binding site on NCAM led to an inhibition of NCAMdependent neurite outgrowth of hippocampal neurons.

NCAM stimulation leads to recruitment of NCAM to lipid rafts (Fig. 10), which is required for NCAM-stimulated neurite outgrowth and depends on palmitoylation of NCAM, and 
triggers NCAM-mediated signaling via the fyn/fak pathway (Niethammer et al., 2002). Mutation of the calmodulin binding motif that overlaps with the palmitoylation sites has no significant effect on cell surface expression, palmitoylation, and raft localization of this mutated NCAM. Furthermore, activation of fyn is not impaired when the calmodulin binding site on NCAM is mutated. However, activation of fak was abolished by mutated NCAM upon NCAM stimulation, indicating that the binding of calmodulin to NCAM is required for the activation of fak.

Disruption of the calmodulin binding to NCAM, which is required for neurite outgrowth, also caused morphological alterations upon NCAM stimulation as seen by spine-like extensions from neurites of neurons expressing mutated NCAM. Since calmodulin cannot bind to mutated NCAM to process the signals triggered by NCAM stimulation, it is plausible to assume that the interaction of calmodulin with cytoskeleton-associated proteins is sustained, leading to undirected and uncontrolled neurite outgrowth induced by ongoing NCAM stimulation and resulting in the formation of abnormal morphological structures.

\section{Binding of calmodulin to NCAM is required for the activation of fak and for the proteolytic processing of fak and NCAM}

NCAM associates with fak as seen by coimmunoprecipitation (Beggs et al., 1997) and NCAM stimulation leads to fak activation (Fig. 10), which is required for NCAM-mediated neurite outgrowth (Niethammer et al., 2002). We showed that disruption of the calmodulin binding to NCAM by mutation abrogated fak activation, but not fyn activation upon NCAM stimulation. In parallel to fak activation, proteolytic processing of fak takes place upon NCAM stimulation (Fig. 10). The cleavage of fak leads to the generation of a C-terminal fragment containing the focal adhesion targeting (FAT) domain, which mediates the localization of fak to focal adhesion sites (Hildebrand et al., 1993) and a noncatalytic N-terminal fragment containing an amino acid sequence that shares homology with the FERM (band 4.1, ezrin, radixin, and moesin) domain, which regulates fak activity and signaling (Cooper et al., 2003; Dunty et al., 2004).

NCAM stimulation leads to the generation of NCAM fragments (Fig. 10): A soluble $55 \mathrm{kDa}$ fragment was recognized in the cell culture supernatant, while a fragment of $50 \mathrm{kDa}$ was detected in cell lysates and in nuclei by an antibody directed against the intracellular membrane-proximal sequence of NCAM and, interestingly, by an antibody against the extracellular domain of NCAM. When the calmodulin binding motif was mutated, the 50 $\mathrm{kDa}$ fragment was not generated, indicating that the binding of calmodulin is required for this cleavage of NCAM triggered by NCAM stimulation. Since neither the $\gamma$-secretase inhibitor DAPT nor the metalloprotease inhibitor GM6001 inhibited the
NCAM-stimulated generation of the intracellular NCAM fragment, we conclude that neither $\gamma$-secretase nor ADAM17/ TACE is responsible for the production of this fragment. However, the serine protease inhibitor aprotinin inhibits the generation of this fragment. Since aprotinin efficiently inhibits the protease plasmin and since it has been shown that NCAM is cleaved by plasmin, resulting in the generation of a fragment with an apparent molecular weight of $\sim 60 \mathrm{kDa}$ (Endo et al., 1998, 1999), it is likely that plasmin cleaves the extracellular domain of NCAM (Fig. 10) and, thus, is the protease responsible for the generation of the $50 \mathrm{kDa}$ NCAM fragment observed in this study.

\section{NCAM stimulation leads to nuclear transport of a transmembrane NCAM fragment and a fak fragment}

An intriguing finding of our study is that the proteolytic processing of NCAM and fak is accompanied by a nuclear import of a $\mathrm{N}$-terminal fak fragment and a C-terminal fragment of NCAM that comprises the intracellular and transmembrane domain as well as part of the extracellular domain (Fig. 10). The generation of both fragments depends on homophilic NCAM transinteraction leading to cis dimerization or oligomerization of NCAM (Fig. 10) and requires the calmodulin binding site in the intracellular domain of NCAM, indicating that the interaction between calmodulin and NCAM is required for the proteolytic 
processing. Nuclear import of a fak fragment has been reported to occur upon stimulation of distinct signal transduction pathways (Lobo and Zachary, 2000; Stewart et al., 2002; Jones and Stewart, 2004). Nuclear translocation of NCAM and fak fragments may represent an alternative or parallel signal pathway that functions in concert with or distinct from the known NCAMinduced pathways to induce cellular responses and/or to make NCAM's signal transduction distinct in ontogenetic development, regeneration, and synaptic plasticity.

Homophilic NCAM interaction and/or clustering of NCAM at the cell surface as well as the interaction of NCAM with calmodulin triggers proteolytic processing of fak and NCAM by yet unknown mechanisms, which may well depend on known and common signal transduction pathways, but will be difficult to dissect since the intracellular signaling network is dynamically interconnected. We showed that the C-terminal NCAM fragment is translocated from the cell surface to the ER after internalization triggered by NCAM stimulation (Fig. 10). Trafficking of proteins from the cell surface to the ER via the endosome has been shown only for few proteins, e.g., certain toxins, SV40 virus, and the EGF receptor (for references, see Liao and Carpenter, 2007). It has been shown that the transmembrane EGF receptor is translocated from the ER membrane to the cytoplasm by an unknown mechanism that involves the sec61 translocon. We observed that the NCAM fragment is also translocated to the ER, but its translocation from the ER membrane to the cytoplasm depends on calmodulin and calcium and not sec61 (Fig. 10). The import of the NCAM fragment from the cytoplasm into the nucleus is mediated by calmodulin and is calcium dependent (Fig. 10). Calmodulin could be bound to the NCAM fragment, which may be associated with the N-terminal fak fragment during import into the nucleus. The mechanism by which calmodulin mediates the transport of proteins through the nuclear pores is not known.

In the nucleus, the NCAM fragment may interact, for instance, with transcription factors and components of the chromatin matrix to influence NCAM-dependent functions in the nervous system, such as synaptic plasticity. Analysis of the multiple actions of NCAM and its nuclear NCAM fragment during the different states of nervous system development and functioning in the adult will thus be an important yet difficult task for future investigations. In particular, investigations on the calmodulin-mediated translocation from the ER to the cytoplasm and nuclear import should yield further insights into a possibly central role of NCAM in crucial nervous system functions. In addition, since increasing evidence indicates that NCAM is related to psychiatric and neurodegenerative disorders, such as schizophrenia and bipolar disorders (for a recent review, see Brennaman and Maness, 2010), investigation of the functional consequences of the import of NCAM fragments into the nucleus should provide new insights into an understanding of distinct molecular mechanisms underlying the diseases and into designing strategies for therapy of the affected neuropsychiatric disorders.

\section{References}

Beggs HE, Soriano P, Maness PF (1994) NCAM-dependent neurite outgrowth is inhibited in neurons from Fyn-minus mice. J Cell Biol 127:825-833.

Beggs HE, Baragona SC, Hemperly JJ, Maness PF (1997) NCAM140 interacts with the focal adhesion kinase p125(fak) and the SRC-related tyrosine kinase p59(fyn). J Biol Chem 272:8310-8319.

Bodrikov V, Leshchyns'ka I, Sytnyk V, Overvoorde J, den Hertog J, Schachner M (2005) RPTPalpha is essential for NCAM-mediated p59fyn activation and neurite elongation. J Cell Biol 168:127-139.
Brennaman LH, Maness PF (2010) NCAM in neuropsychiatric and neurodegenerative disorders. Adv Exp Med Biol 663:299-317.

Conboy L, Bisaz R, Markram K, Sandi C (2010) Role of NCAM in emotion and learning. Adv Exp Med Biol 663:271-296.

Cooper LA, Shen TL, Guan JL (2003) Regulation of focal adhesion kinase by its amino-terminal domain through an autoinhibitory interaction. Mol Cell Biol 23:8030-8041.

Cremer H, Lange R, Christoph A, Plomann M, Vopper G, Roes J, Brown R, Baldwin S, Kraemer P, Scheff S, Barthels D, Rajewsky K, Wille W (1994) Inactivation of the N-CAM gene in mice results in size reduction of the olfactory bulb and deficits in spatial learning. Nature 367:455-459.

Cunningham BA, Hoffman S, Rutishauser U, Hemperly JJ, Edelman GM (1983) Molecular topography of the neural cell adhesion molecule NCAM: surface orientation and location of sialic acid-rich and binding regions. Proc Natl Acad Sci U S A 80:3116-3120.

Ditlevsen DK, Kolkova K (2010) Signaling pathways involved in NCAMinduced neurite outgrowth. Adv Exp Med Biol 663:151-168.

Ditlevsen DK, Povlsen GK, Berezin V, Bock E (2008) NCAM-induced intracellular signaling revisited. J Neurosci Res 86:727-743.

Dityateva G, Hammond M, Thiel C, Ruonala MO, Delling M, Siebenkotten G, Nix M, Dityatev A (2003) Rapid and efficient electroporation-based gene transfer into primary dissociated neurons. J Neurosci Methods 130:65-73.

Dunty JM, Gabarra-Niecko V, King ML, Ceccarelli DF, Eck MJ, Schaller MD (2004) FERM domain interaction promotes FAK signaling. Mol Cell Biol 24:5353-5368.

Endo A, Nagai N, Urano T, Ihara H, Takada Y, Hashimoto K, Takada A (1998) Proteolysis of highly polysialylated NCAM by the tissue plasminogen activator-plasmin system in rats. Neurosci Lett 246:37-40.

Endo A, Nagai N, Urano T, Takada Y, Hashimoto K, Takada A (1999) Proteolysis of neuronal cell adhesion molecule by the tissue plasminogen activator-plasmin system after kainate injection in the mouse hippocampus. Neurosci Res 33:1-8.

Füllekrug J, Simons K (2004) Lipid rafts and apical membrane traffic. Ann NY Acad Sci 1014:164-169.

Gennarini G, Rougon G, Deagostini-Bazin H, Hirn M, Goridis C (1984) Studies on the transmembrane disposition of the neural cell adhesion molecule N-CAM. A monoclonal antibody recognizing a cytoplasmic domain and evidence for the presence of phosphoserine residues. Eur J Biochem 142:57-64.

Hildebrand JD, Schaller MD, Parsons JT (1993) Identification of sequences required for the efficient localization of the focal adhesion kinase, pp125FAK, to cellular focal adhesions. J Cell Biol 123:993-1005.

Jones G, Stewart G (2004) Nuclear import of N-terminal FAK by activation of the FcepsilonRI receptor in RBL-2H3 cells. Biochem Biophys Res Commun 314:39-45.

Kalus I, Schnegelsberg B, Seidah NG, Kleene R, Schachner M (2003) The proprotein convertase PC5A and a metalloprotease are involved in the proteolytic processing of the neural adhesion molecule L1. J Biol Chem 278:10381-10388.

Kalus I, Bormann U, Mzoughi M, Schachner M, Kleene R (2006) Proteolytic cleavage of the neural cell adhesion molecule by ADAM17/TACE is involved in neurite outgrowth. J Neurochem 98:78-88.

Kiselyov VV (2010) NCAM and the FGF-receptor. Adv Exp Med Biol 663:67-79.

Kolkova K, Novitskaya V, Pedersen N, Berezin V, Bock E (2000) Neural cell adhesion molecule-stimulated neurite outgrowth depends on activation of protein kinase $\mathrm{C}$ and the Ras-mitogen activated protein kinase pathway. J Neurosci 20:2238-2246.

Lee JL, Wang MJ, Chen JY (2009) Acetylation and activation of STAT3 mediated by nuclear translocation of CD44. J Cell Biol 185:949-957.

Liao HJ, Carpenter G (2007) Role of the Sec61 translocon in EGF receptor trafficking to the nucleus and gene expression. Mol Biol Cell 18:1064-1072.

Little EB, Edelman GM, Cunningham BA (1998) Palmitoylation of the cytoplasmic domain of the neural cell adhesion molecule N-CAM serves as an anchor to cellular membranes. Cell Adhes Commun 6:415-430.

Lobo M, Zachary I (2000) Nuclear localization and apoptotic regulation of an amino-terminal domain focal adhesion kinase fragment in endothelial cells. Biochem Biophys Res Commun 276:1068-1074.

Loers G, Chen S, Grumet M, Schachner M (2005) Signal transduction path- 
ways implicated in neural recognition molecule L1 triggered neuroprotection and neuritogenesis. J Neurochem 92:1463-1476.

Makhina T, Loers G, Schulze C, Ueberle B, Schachner M, Kleene R (2009) Extracellular GAPDH binds to L1 and enhances neurite outgrowth. Mol Cell Neurosci 41:206-218

Maness PF, Schachner M (2007) Neural recognition molecules of the immunoglobulin superfamily: signaling transducers of axon guidance and neuronal migration. Nat Neurosci 10:19-26.

Muller D, Mendez P, Deroo M, Klauser P, Steen S, Poglia L (2010) Role of NCAM in spine dynamics and synaptogenesis. Adv Exp Med Biol 663:245-256.

Niethammer P, Delling M, Sytnyk V, Dityatev A, Fukami K, Schachner M (2002) Cosignaling of NCAM via lipid rafts and the FGF receptor is required for neuritogenesis. J Cell Biol 157:521-532.

Norman JA, Ansell J, Stone GA, Wennogle LP, Wasley JW (1987) CGS 9343B, a novel, potent, and selective inhibitor of calmodulin activity. Mol Pharmacol 31:535-540.

Oppizzi ML, Akhavan A, Singh M, Fata JE, Muschler JL (2008) Nuclear translocation of beta-dystroglycan reveals a distinctive trafficking pattern of autoproteolyzed mucins. Traffic 9:2063-2072.
Paratcha G, Ledda F, Ibáñez CF (2003) The neural cell adhesion molecule NCAM is an alternative signaling receptor for GDNF family ligands. Cell 113:867-879.

Planque N (2006) Nuclear trafficking of secreted factors and cell-surface receptors: new pathways to regulate cell proliferation and differentiation, and involvement in cancers. Cell Commun Signal 4:7.

Ponimaskin E, Dityateva G, Ruonala MO, Fukata M, Fukata Y, Kobe F, Wouters FS, Delling M, Bredt DS, Schachner M, Dityatev A (2008) Fibroblast growth factor-regulated palmitoylation of the neural cell adhesion molecule determines neuronal morphogenesis. J Neurosci 28:8897-8907.

Rhoads AR, Friedberg F (1997) Sequence motifs for calmodulin recognition. FASEB J 11:331-340.

Rolf B, Lang D, Hillenbrand R, Richter M, Schachner M, Bartsch U (2003) Altered expression of CHL1 by glial cells in response to optic nerve injury and intravitreal application of fibroblast growth factor-2. J Neurosci Res 71:835-843.

Stewart A, Ham C, Zachary I (2002) The focal adhesion kinase aminoterminal domain localises to nuclei and intercellular junctions in HEK 293 and MDCK cells independently of tyrosine 397 and the carboxyterminal domain. Biochem Biophys Res Commun 299:62-73.

Wagstaff KM, Jans DA (2009) Importins and beyond: non-conventional nuclear transport mechanisms. Traffic 10:1188-1198. 